\title{
Die vielen Wege zur Effektuierung des Völkerrechts
}

\author{
Anne van Aaken"
}

A. Einleitung

B. Theorienpluralismus zur Erklärung der

Befolgung des Völkerrechts .......... 230

I. Definition(en) der Befolgung des Völkerrechts

II. Rationale Wahl vs. Konstruktivismus?

III. Reziprozität, Reputation und Retaliation: die Problemfelder.......... 239

C. Klassische Mechanismen zur Effektuierung des Völkerrechts 244

I. Zentrale Durchsetzung des Völkerrechts ........................... 244

II. Dezentrale Durchsetzung auf internationaler Ebene................ 246

1. Internationale Streitbeilegungsmechanismen ................... 246

a) Außergerichtliche Streitbeilegungsmechanismen 246 b) Zwischenstaatliche gerichtliche Verfahren ............... 247

c) Verfahren mit Individuen ... 249

2. Monitoring und Reporting ...... 251

3. Staatenverantwortlichkeit...... 252

4. Verlinkung von Verträgen....... 253

III. Dezentrale Durchsetzung auf nationaler Ebene ........................ 254

1. Nationale Gerichte............ 254

2. Nationale politische Prozesse.... 255

D. Transnationale Mechanismen zur Effektuierung des Völkerrechts ....... 257

I. Nutzung des Marktmechanismus:

Konsumentenpräferenzen .......... 258

II. Marktmechanismus: Netzwerkexternalitäten ...................... 260

E. Ausblick: Eine Skizze von Bauprinzipien zur Effektuierung des Völkerrechts

\section{A. Einleitung}

Traditionell haben Staaten Völkerrecht sowohl gesetzt, als auch durchgesetzt. Dies hat sich geändert: Einerseits sind mehr Akteure auf die Bühne der Durchsetzung getreten, andererseits wird Völkerrecht heute auch über vielfältigere Mechanismen durchgesetzt. Zentral für die Problemlösungskapazität des Rechts ist dessen Durchsetzungsfähigkeit; eine Eigenschaft, die beim dezentralen und konsensual geprägten Völkerrecht öfters angezweifelt wird. Sowohl Rechtswissenschaftler als auch Politikwissenschaftler zeigen daher seit langer Zeit Interesse an Befolgungsfragen. Erst seit ca. 15 Jahren allerdings werden diese Fragen ausführlich und detailliert behandelt, insbesondere in empirischer Hinsicht. ${ }^{1}$ Dabei standen sich lange Zeit zwei Annahmen gegenüber: die implizite (optimistische rechtswissenschaftliche), dass Staaten das Völkerrecht in aller Regel freiwillig befolgen und die explizite (skeptische politikwissenschaftliche), dass Völkerrecht ein reines Epiphänomen ist. Diese Auffassungen sind in ihrer reinen Form nicht mehr vertretbar. Stattdessen werden nun im Detail Kausalmechanismen gesucht, aufgrund derer Völkerrecht effektuiert wird, denn nur wenn Wissen darüber vorhanden ist, warum und unter welchen Umständen Völkerrecht befolgt wird, kann es auch effektiver gestaltet werden. Neben das Völ-

* Prof. Dr. Anne van Aaken hat den Lehrstuhl für Law and Economics, Rechtstheorie, Völker- und Europarecht an der Universität St. Gallen. Ich danke Simone Beckers für exzellente redaktionelle Hilfe.

1 J.L. Dunhoff/M.A. Pollack, Reviewing two Decades of IL/IR Scholarship, in: J.L. Dunhoff/M.A. Pollack (Hrsg.), Interdisciplinary Perspectives on International Law and International Relations. The State of the Art, Cambridge: Cambridge University Press 2013, S. 626 (638). 
kerrecht sind - teilweise in Verbindung mit demselben - soft law sowie „Transnationale Private Regulierung “ (TRP) $)^{2}$ getreten, die teilweise Defizite von Normen ausgleichen sowie deren Durchsetzung ergänzen. Auch sie werden nun vermehrt analysiert. Auf Grundlage dieser Untersuchungen können die jeweils adäquaten Institutionen gewählt werden, die eine Effektuierung des Völkerrechts versprechen.

Dieser Artikel versteht sich als interdisziplinärer Beitrag zur Analyse der verschiedenen Durchsetzungsmechanismen des Völkerrechts. ${ }^{3}$ Dabei steht die Frage, warum und unter welchen Umständen Staaten sich an Völkerrecht halten im Vordergrund; aber auch neuartige Durchsetzungsmechanismen und ihre Interaktion mit dem Völkerrecht werden betrachtet. Diese Fragen lassen sich nur mittels sozialwissenschaftlicher Methoden beantworten. ${ }^{4}$ Diese Methoden indes bedürfen ihrerseits des Rechts, um Antworten liefern zu können, denn Normen und Institutionen geben den Rahmen vor, in welchem sich Staaten (und andere Akteure) bewegen. In Bezug auf die Verhaltenswirkungen des Rechts, steckt der Teufel, wie zumeist, in den institutionellen und damit rechtlichen Details.

Der Artikel verwendet eine Typologie von faktischen Problemstrukturen (und damit Anreizstrukturen), die es erlaubt, institutionelle Bauprinzipien zu erarbeiten, welche zur Effektuierung des Völkerrechts herangezogen werden können. So werden sowohl

2 Soft law wird hier gebraucht für alle transnationale Normen (ungeachtet des Akteurs, der sie generiert), die nicht den Völkerrechtsquellen gemäß Art. 38 IGH Statut entsprechen. Vgl. überblickshalber D. Thürer, Soft Law, in: R. Bernhardt (Hrsg.), Encyclopedia of Public International Law, Vol. 4, Amsterdam: Elsevier 2000, S. 452. Eine andere, funktionale Definition von Soft Law, wird von den Politikwissenschaftlern K.W. Abbott/D. Snidal, Hard and Soft Law in International Governance, 54 International Organization 2000, S. 421, verwendet, die anhand dreier Kriterien die "Weichheit" einer Norm auf einem Kontinuum abbilden: Verpflichtung (obligation), Präzision (precision) und Delegation (delegation). Hartes Recht meint rechtlich bindendes Recht, welches präzise ist und durch eine Drittpartei interpretiert und durchgesetzt wird. Dem folgend G.C. Shaffer/M.A. Pollack, Hard Law and Soft Law, in: J.L. Dunhoff/M.A. Pollack (Hrsg.), Perspectives (Fn. 1), S. 197. Noch anders J. Pauwelyn, Informal International Public Policy Making (IIPPM), Project Framing Paper für HiiL, 31. Mai 2010 (erhältlich bei der Autorin), S. 9-10: "Cross-border cooperation between public authorities, with or without the participation of private actors and/or international organizations, in a forum other than a traditional international organization (process informality), and/or as between actors other than traditional diplomatic actors (such as regulators or agencies) (actor informality), and/or which does not result in a formal treaty or legally enforceable commitment (output informality)."

Transnational Private Regulation umfasst auch hybride Regulierung durch NGOs, Unternehmen(-sverbände), Staaten, und IGOs, die auf Markt- und Staatsversagen auf der globalen Ebene antworten, Risiko managen und globale öffentliche Güter regulieren. Vgl. umfassend dazu C. Scott/F. Cafaggi/L. Senden (Hrsg.), The Challenges of Transnational Private Regulation: Conceptual and Constitutional Debates, Oxford et al.: Blackwell 2011. Zur Durchsetzung siehe F. Cafaggi (Hrsg.), The Enforcement of Transnational Regulation. Ensuring Compliance in a Global World, Cheltenham: Edward Elgar 2012.

3 Aus rechtswissenschaftlicher Sicht übersichtshalber statt vieler, siehe $S$. Vöneky, Die Durchsetzung des Völkerrechts, Jura 2007, S. 488 sowie A. v. Arnauld, Völkerrecht, Heidelberg 2012, S. 172 ff.

4 Nicht speziell behandelt werden soll die Debatte über die Konstitutionalisierung und (materiell-rechtliche) Hierarchisierung des Völkerrechts, da sich die Durchsetzungsmechanismen an sich nicht geändert haben (auch wenn es eine Proliferation internationaler Gerichte gibt). Übersichtshalber siehe J. Klabbers/ A. Peters/G. Ulfstein, The Constitutionalization of International Law, Oxford: Oxford University Press 2011 sowie A. Peters, Global Constitutionalism in a Nutshell, in: K. Dicke/S. Hobe/K.-U. Meyn/A. Peters/E. Riedel/H.-J. Schütz/C. Tietje (Hrsg.), Weltinnenrecht: Liber amicorum Jost Delbrück, Berlin 2005, S. 535. 
auf Reziprozität ${ }^{5}$ beruhende internationale Verträge, wie sie z.B. im Handelsrecht Anwendung finden, herangezogen, als auch Verträge, die öffentliche Güter schützen bzw. bereitstellen (etwa Menschenrechtsverträge oder Umweltverträge) und die eher mit Reputationsmechanismen arbeiten müssen.

Die sozialwissenschaftlichen Theorien, die bei der Definition von Problemstrukturen und bei der Erarbeitung der adäquaten Mechanismen (Lösungsstrukturen) als Hilfen dienen, bilden keine homogene Gruppe. Hier soll nicht eine Einheitstheorie Erklärungen bieten, vielmehr wird hinterfragt, welchen Mehrwert jede einzelne Theorie zur Erklärung des Phänomens der Effektuierung des Völkerrechts zu liefern vermag. Eine Methodenorthodoxie wird nicht vertreten. Als Ausgangspunkt wird die Theorie der rationalen Wahl verwendet, sie wird sodann angereichert durch andere Theorien. Eine Zusammenschau durch alle Theorien informiert im Anschluss über die praktische Wirksamkeit des Völkerrechts (B.).

Völkerrecht ist herkömmlich und primär ein Recht zwischen Staaten (und internationalen Organisationen, IGOs), selbst wenn die geregelten Sachverhalte innerstaatliche Konstellationen betreffen bzw. bei Umsetzung und Effektuierung letztendlich nationale Rechtsunterworfene (Individuen oder Unternehmen) die Adressaten sind (was nicht notwendig identisch ist mit der Schutzrichtung des Rechts). Eine Betrachtung nur der zwischenstaatlichen Ebene mit dem Staat als „black box“ bleibt wichtig, würde aber zu kurz greifen zur Erklärung von Effektuierungsmechanismen in bestimmten Bereichen: Völkerrecht wird auch innerstaatlich effektuiert. Erklärungen zur Befolgung des Völkerrechts bedürfen zumindest der „Zwei-Ebenen-Analyse“ (two-level-game) ${ }^{6}$ (C.).

Weiterhin existieren vermehrt transnationale Mechanismen zur Effektuierung des Völkerrechts und des soft laws. Zunehmend werden TPR und Marktmechanismen genutzt, bei denen nichtstaatliche Akteure wie Unternehmen und Nichtregierungsorganisationen (NGOs) eine wichtige Rolle einnehmen. Diese sind maßgeblich an der Durchsetzung von Völkerrechtsnormen und soft law beteiligt. Bislang weitgehend theoretisch vernachlässigt sind die Nutzung von Netzwerkexternalitäten sowie solche Marktmechanismen, die über Konsumentenpräferenzen andere nichtstaatliche Akteure zur Befolgung des Völkerrechts oder soft laws anhalten können in einer Weise, die staatlichen Institutionen verwehrt ist (D.).

5 Rechtstheoretisch dazu V. Boehme-Neßler, Reziprozität und Recht, 39 Rechtstheorie 2008, S. 521; aus völkerrechtlicher Sicht grundlegend B. Simma, Das Reziprozitätselement im Zustandekommen völkerrechtlicher Verträge. Gedanken zu einem Bauprinzip der internationalen Rechtsbeziehungen, Berlin 1972 sowie B. Simma, Das Reziprozitätselement in der Entstehung des Völkergewohnheitsrechts, München 1970.

6 R.D. Putnam, Diplomacy and Domestic Politics: The Logic of Two-Level Games, 42 International Organization 1988, S. 427. 
Aufbauend auf den vorangegangen Teilen skizziert der letzte Teil Bauprinzipien, die jeweils im Hinblick auf bestimmte Problemstrukturen des Völkerrechts eine Effektuierung erwarten lassen. Je nach Problemstruktur werden für die beteiligten Akteure andere Anreize gesetzt, daher bedürfen sie auch anderer institutioneller, d.h. rechtlicher, Lösungen. Hieraus können Erkenntnisse über das passende Vertragsdesign gewonnen werden, also darüber, in welchen Situationen welche Durchsetzungsmechanismen Erfolg versprechen (E.).

\section{B. Theorienpluralismus zur Erklärung der Befolgung des Völkerrechts}

Ob und warum Völkerrecht befolgt wird (oder nicht), hat die besten Köpfe aus den unterschiedlichsten Disziplinen beschäftigt. Folgende Beobachtung von Louis Henkin wird häufig zitiert: "almost all nations observe almost all principles of international law and almost all of their obligations almost all the time."7 Allerdings ist fraglich, ob diese Aussage korrekt ist und warum und unter welchen Umständen Nationen die Prinzipien des Völkerrechts befolgen. Es bedarf insofern einer Theorie darüber, wie Völkerrecht in den internationalen Beziehungen wirkt, sei es im Hinblick auf resistente oder kooperative Staaten. Davon könnten sowohl die Politik (für das Design von Verträgen) als auch die (internationalen) Gerichte profitieren, welche die Frage zu beantworten haben, welche Auslegung das Völkerrecht am besten effektuiert (effet utile im weiteren Sinne). Die zugrundeliegenden Annahmen sowie die Methoden dazu variieren beträchtlich. Eine umfassende Analyse würde den Umfang des Artikels überschreiten, aber ein kurzer Abriss über die theoretischen Ansätze ist notwendig, um die vielen Mechanismen zur Effektuierung des Völkerrechts einordnen und als Bauprinzipien identifizieren zu können.

\section{Definition(en) der Befolgung des Völkerrechts}

Schon das Zitat von Henkin illustriert die Ambiguität des Ausdrucks „observe“. Ist hier nur normkonformes Verhalten gemeint oder insinuiert Henkin eine Kausalität des Rechts für die Normbefolgung? Ein Beispiel veranschaulicht diese Frage: Die Einhaltung von internationalen Menschenrechtsverträgen kann darauf beruhen, dass dieselben Rechte bereits durch das nationale Recht geschützt sind und die Ratifizierung eines Vertrages daher für die Menschenrechtslage in diesem Land keinen Unterschied mehr macht. ${ }^{8}$ Dann wäre aber der internationale Vertrag an sich überflüssig. In diesem Sinne hielt sich Russland (aufgrund der Rezession) zur Zeit des Beitritts bereits an das Kyoto-Protokoll; die ökonomischen Umstände, nicht das Kyoto-Pro-

7 L. Henkin, How Nations Behave, London: Pall Mall Press 1968, S. 47.

8 B. Simmons, Mobilizing for Human Rights: International Law in Domestic Politics, Cambridge: Cambridge University Press 2009, S. 150 ff. 
tokoll, führten zur Normbefolgung seitens Russlands. ${ }^{9}$ Kann der Nichteinmarsch Iraks in die Türkei (im Gegensatz zu Kuwait) als Regelbefolgung angesehen werden?

Zur Beantwortung der Frage, ob Völkerrecht (oder soft law) verhaltenswirksam ist, muss ein Unterschied gemacht werden zwischen Effektivität (effectiveness) und Befolgung (compliance). Während Effektivität eine Kausalität zwischen Norm und Verhalten misst, bedeutet Befolgung einfach nur eine Korrelation zwischen Norm und Verhalten. Ob die Norm kausal war für das normkonforme Verhalten ist dabei unbeachtlich. ${ }^{10}$ Davon zu unterscheiden ist nochmals die Vertragseffektivität in dem Sinne, dass trotz Befolgung des Rechts durch die Staaten der Vertrag nicht effektiv ist: dass also seine Ziele ${ }^{11}$ trotz Einhaltung nicht erreicht werden, etwa wenn sich trotz befolgter Fischfangquoten eine Spezies nicht mehr auf eine nachhaltige Anzahl vermehren kann. ${ }^{12}$

Effektuierungsfragen konzentrieren sich auf das Problem, ob Völkerrecht verhaltenswirksam ist. Sie sind deshalb besonders spannend, weil das Völkerrecht - anders als das nationale Recht - keine obligatorische Gerichtsbarkeit kennt, keine Weltpolizei und keinen Gerichtsvollzieher. Es ist kein Subordinationsrecht, sondern beruht auf der souveränen Gleichheit der Staaten, ${ }^{13}$ was manche dazu bewogen hat, dem Völkerrecht seine Rechtsqualität abzusprechen. ${ }^{14}$ Dies verkennt aber die vielfältigen Wirkungsweisen des Völkerrechts, die hier aufgezeigt werden sollen. ${ }^{15}$

9 A. Bernard/S. Paltsev/J.M. Reilly/M. Vielle/L. Viguier, Russia's Role in the Kyoto Protocol, MIT Joint Program on the Science and Policy of Global Change, Report No. 98, 2003, S. 3 f., erhältlich unter: <http://globalchange.mit.edu/files/document/MITJPSPGC_Rpt98.pdf> (zuletzt besucht am 15. Mai 2013).

10 Zu dieser Unterscheidung, siehe A.T. Guzman, A Compliance-Based Theory of International Law, 90 California Law Review 2002, S. 1823, A. T. Guzman, How International Law Works: A Rational Choice Theory, Oxford: Oxford University Press 2008, S. 22 f. Vgl. zur Definitionsproblematik von Compliance auch J. v. Stein, The Engines of Compliance, in: J.L. Dunhoff/M.A. Pollack (Hrsg.), Perspectives (Fn. 1), S. 477 (493 ff.) sowie K. Raustiala/A.-M. Slaughter, International Law, International Relations and Compliance, in: W. Carlsnaes/T. Risse/B.A. Simmons (Hrsg.), Handbook of International Relations, London: Sage Publication 2001, S. 538.

11 Im Sinne von Art. 31 Abs. 1 Wienervertragsrechtskonvention.

12 Vgl. dazu A. Chayes/A. Handler Chayes, On Compliance, 47 International Organization 1993, S. 175 (176, Fn. 3).

13 Vgl. I. Brownlie, Principles of Public International Law, Oxford: Oxford University Press 2003, S. 33 f. sowie S. Vöneky, Durchsetzung (Fn. 3), S. 488 (488).

14 J. Austin, Lectures on Jurisprudence, Bd. I, London: John Murray 1885, Lecture I, S. 86 ff., Lecture V, S. 172, sprach dem Völkerrecht seine Rechtsqualität ab, da Recht mit der Durchsetzung von Geboten und Verboten im Wege des Zwanges und der Sanktion für die Missachtung von Sollenssätzen verknüpft sei. Vgl. hierzu auch rechtstheoretisch ausführlich H.C Röhl/K.F. Röhl, Allgemeine Rechtslehre, München 2008, S. $314 \mathrm{ff}$.

15 Siehe etwa A. D’Amato, International Law: Process and Prospect, New York: Transnational 1997, S. $50 \mathrm{f}$.: "(T)he absence of these institutions does not mean that international law is not really law; rather, it simply means that international law is enforced in a different way." "Recht lässt sich gegen Ungehorsam letztlich nur mit Zwang durchsetzen.... Recht ohne Zwang ist nicht denkbar.... Das Völkerrecht, das vielfach nicht durchsetzbar ist, nimmt eine Sonderstellung ein”, so G. Arzt, Einführung in die Rechtswissenschaft, Basel 1987, S. 40. 
Ein weiteres Problem ergibt sich daraus, dass aufgrund des konsensualen Charakters des Völkerrechts Rechtssetzer und Rechtsunterworfene - im Unterschied zu nationalem Recht - identisch sind. Dies ist insbesondere für die Frage des Völkergewohnheitsrechts relevant. Stellt eine Abweichung von der geltenden Norm eine Weiterentwicklung des Rechts (Übungskomponente) dar oder aber dessen Bruch? ${ }^{16}$ Hilfreich erscheint in diesem Zusammenhang eine Unterscheidung aus der Institutionenökonomik. Dort wird zwischen Regelgeltungsinteresse einerseits und Regelbefolgungsinteresse andererseits differenziert. Während mangelndes Regelbefolgungsinteresse die Wirksamkeit von Rechtsnormen (d.h. die Effektivität) beeinträchtigt, gefährdet mangelndes Regelgeltungsinteresse (Akzeptanz der Rechtsnormen) die Stabilität von Rechtsordnungen insgesamt, oder, im Falle vom Völkerrecht, die Stabilität eines Vertrages bzw. des Gewohnheitsrechts, da immerzu auf die Änderung der Rechtsregeln gedrängt wird. ${ }^{17}$ Im Nicaragua Fall argumentierte der IGH, dass es für Völkergewohnheitsrecht ausreicht, wenn das Verhalten von Staaten im Allgemeinen normkonform ist, sofern Abweichungen von der Norm nur "as breaches of that rule, not as indications of the recognition of a new rule" angesehen werden. ${ }^{18}$ Damit wird bereits klar, dass im Falle des Völkergewohnheitsrechts nicht erforderlich ist, dass sich alle Staaten immer daran halten. Übung beinhaltet auch Regelbruch, solange dieser als solcher von anderen Staaten betrachtet wird (opinio iuris). ${ }^{19}$ Rechtstheoretisch gilt dasselbe für Völkervertragsrecht. Völkerrechtsbrüche gefährden die Rechtsordnung an sich erst einmal nicht, solange sie, wenn auch nur auf diplomatischem Wege (etwa durch Protestnote, ohne Sanktion des Rechtsbruches)

16 Ausführlich A.E. Roberts, Traditional and Modern Approaches to Customary International Law, 95 American Journal of International Law 2001, S. 757.

17 Deutlich wird dieser Unterschied bei einem Beispiel von V. Vanberg, Die Akzeptanz von Institutionen, in: W. Korff (Hrsg.), Handbuch der Wirtschaftsethik, Gütersloh 1999, S. 38: Der Dieb hat kein Regelbefolgungsinteresse, sehr wohl aber ein Regelgeltungsinteresse, während der Revolutionär kein Regelgeltungsinteresse hat. Ähnlich A. Chayes/A. Handler Chayes, Compliance (Fn. 12), S. 183 f.: "It is true that a state's incentive at the treaty negotiation stage may be different from those it faces when the time for compliance rolls around.... Nevertheless, the very act of making commitments embodied in an international agreement changes the calculus at the compliance stage, if only because it generates expectations of compliance in others that must enter into the equation".

18 Nicaragua, 1986 ICJ Rep. S. 98, Rz. 186.

19 Der Stellenwert der opinio iuris zur Bildung von Gewohnheitsrecht ist umstritten. Vgl. D. Vagts, International Relations Looks at Customary International Law: A Traditionalist's Defense, 15 European Journal of International Law 2004, S. 1031. R. Jennings, What is International Law and How do We Tell It, When We See It?, 37 Schweizerisches Jahrbuch für Internationales Recht 1981, S. 59 (65-71); A. d'Amato, The Concept of Custom in International Law, Ithaca/London: Cornell University Press 1971. Er betrachtet die Zirkularität der opinio juris als "fatal defect" (S. 66): "How can Custom create law if its psychological component requires action in conscious accordance with preexisting law?" Ähnlich P. Hulsroj, Three Sources - No River, 54 Zeitschrift für öffentliches Recht (1999), S. 219 (230): "Opinio iuris is thus nothing but a requirement that states must have suffered from a legal misconception when following a practice." Richtigerweise aber m.E. A.E. Roberts, Approaches (Fn. 16). 
als solche erkannt werden; ${ }^{20}$ also das Rechtsgeltungsinteresse mehrheitlich weiter besteht.

Ein weiteres Problem ergibt sich aus der definitorischen Bestimmtheit von „Befolgung “. Völkerrecht verwendet oftmals unbestimmte Rechtsbegriffe, ist mehrdeutig und vage. Dies wiegt besonders schwer, wenn eine autoritative Auslegungsinstanz fehlt. Denn ein Verhalten kann dann, sowohl aufgrund faktischer als auch rechtlicher Unsicherheit, entweder als Rechtsbruch oder aber als rechtskonformes Verhalten betrachtet werden: Befolgung ist daher als Spektrum, nicht als Dichotomie zu verstehen. ${ }^{21}$ Zugleich ergibt sich hier das Problem der Interaktion der zeitlich getrennten Phasen zwischen Vertragsgestaltung und Befolgung. Diese sind nicht unabhängig voneinander, aber aus analytischen Gründen zu trennen. Dieser Artikel konzentriert sich allein auf die Befolgungsphase des Rechts, obschon diese wiederum, wie oben angesprochen, informieren kann über ein "rational design" des Völkerrechts. ${ }^{22}$ Diese Indeterminiertheit des Völkerrechts hat Chayes and Chayes mit ihrem "managerial approach" 23 der Befolgungsfrage dazu bewogen, zwischen "tiefen" regulatorischen Abkommen und generellen Rahmenabkommen zu unterscheiden. Ihre Hypothese lautet, dass Staaten keine Abkommen abschließen, die bereits bei Abschluss ihre Unwirksamkeit in sich tragen, weil die Erwartung des Regelbefolgungsinteresses aller Staaten zu gering ist und damit auch das Regelgeltungsinteresse entfällt. Steht dies zu befürchten, so werden Staaten eher "seichte”, generelle (Rahmen)abkommen mit einer höheren Befolgungswahrscheinlichkeit (aber damit auch mit einer geringeren Verhaltenswirksamkeit) abschließen. ${ }^{24}$

Indeterminiertheit des Rechts ist ein subtiler Flexibilitätsmechanismus, ${ }^{25}$ der es dem einzelnen Staat erlaubt, sich innerhalb des Rechts zu bewegen, während er gleichzeitig für alle Staaten die notwendige Weiterentwicklung des Rechts innerhalb der Normen erlaubt (ohne explizite Vertragsänderungen vornehmen zu müssen). ${ }^{26}$ Kurz-

20 Dieses Problem wurde bereits früh aus soziologischer Sicht behandelt, vgl. T. Geiger, Vorstudien zu einer Soziologie des Rechts, Berlin 1987 (Erstauflage 1947).

21 J. v. Stein, Engines (Fn. 10), S. 478.

22 B. Koremenos/C. Lipson/D. Snidal, The Rational Design of International Institutions, 55 International Organization 2001, S. 761; B. Koremenos/C. Lipson/D. Snidal, Rational Design: Looking Back to Move Forward, 55 International Organization 2001, S. 1051.

23 A. Chayes/A. Handler Chayes, Compliance (Fn. 12), S. 175 sowie A. Chayes/A. Chayes Handler, The New Sovereignty. Compliance with International Regulatory Agreements, Cambridge/Mass.: Harvard University Press 1995.

24 Vgl. G.W. Downs/D.M. Rocke/P.N. Barsoom, Is the Good News about Compliance Good News about Cooperation?, 50 International Organization 1996, S. 379.

25 Anders als etwa Vorbehalte, die explizite Flexibilitätsmechanismen darstellen. E.T. Swaine, Reserving, 31 Yale Journal of International Law, 2006, S. 307, P. Hilpold, Das Vorbehaltsregime der Wiener Vertragskonvention, 34 Archiv des Völkerrechts 1996, S. 376. Weitere Flexibilitätsmechanismen sind etwa die Relegation von Normen, insbes. von technischen Standards, in einfacher zu ändernde Annexe.

26 Daher sind nicht nur Menschenrechtsverträge "living instruments" (für diese siehe EGMR Tyrer $v$. the United Kingdom, Judgment of 25 April 1978, 26 Eur.Ct. H.R (ser. A), S. 15-16, Rz. 31 und nachfolgende Rechtsprechung). 
um, Rechtsbruch ist keine binäre Angelegenheit. Geht es um die Effektuierung des Völkerrechts, so müssen Flexibilitätsmechanismen einbezogen werden. Dasselbe Verhalten eines Staates kann nämlich, je nach Ausgestaltung des Vertrages bzw. je nach seiner Auslegung, einmal zur Völkerrechtswidrigkeit, einmal zur Völkerrechtskonformität führen, je nach Flexibilität des Rechts. ${ }^{27}$ Gibt es keine Auslegungsinstanz, so ist eine Selbstauslegung der Staaten „last resort“, kann aber selbstverständlich „self-serving“ sein. ${ }^{28}$ Jede empirische Untersuchung zur Effektuierung des Völkerrechts hat daher die Flexibilitätsmechanismen (explizite wie Vorbehalte und implizite wie unbestimmte Rechtsbegriffe) mit zu berücksichtigen. ${ }^{29}$

\section{Rationale Wahl vs. Konstruktivismus?}

Die Theorien der internationalen Beziehungen zeigten sich lange skeptisch im Hinblick auf die Wirksamkeit des Völkerrechts. ${ }^{30}$ Die zumeist gegebene Antwort war, dass gerade in puncto Befolgung des Völkerrechts allein Macht und Staateninteressen die internationalen Beziehungen bestimmen. ${ }^{31}$ Die Befolgung von Völkerrecht wurde für geraume Zeit entweder als Funktion von Zwangsausübung durch andere Staaten oder aber als zufälliger Interessenszusammenfall gesehen. ${ }^{32}$ Dies greift aber zu kurz. Mittlerweile ist überwiegend anerkannt, ${ }^{33}$ dass "international law matters". Das Ausmaß dieser Bedeutung allerdings ist weiterhin umstritten. Bei der Frage der Be-

27 Siehe dazu aus der Sicht der ökonomischen Vertragstheorie R.E. Scott/P.B. Stephan, The Limits of Leviathan. Contract Theory and the Enforcement of International Law, Cambridge: Cambridge University Press 2006. Für das Welthandelsrecht S. Schropp, Trade Policy Flexibility and Enforcement in the WTO. A Law and Economics Analysis, Cambridge: Cambridge University Press 2009; für das Investitionsschutzrecht, A.van Aaken, International Investment Law Between Commitment and Flexibility: A Contract Theory Analysis, 12 Journal of International Economic Law 2009, S. 507.

$28 \mathrm{Zu}$ diesem "self-serving bias" in der Verhaltensökonomie, siehe L. Babcock/G. Loewenstein, Explaining Bargaining Impasse: The Role of Self-Serving Biases, 11 Journal of Economic Perspectives 1997, S. 109. Für ein Beispiel vgl. etwa R. Wedgwood, Making a Case for Invading Iraq, The National Law Journal, 30. Oktober 2002, erhältlich unter: http://www.law.com/jsp/article.jsp?

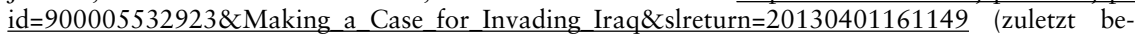
sucht am 15. Mai 2013).

29 Ausführlich nun dazu L.R. Helfer, Flexibility in International Agreements, in: J.L. Dunoff/M.A. Pollack (Hrsg.), Perspectives (Fn. 1), S. 175.

30 Vgl. aber nun die vielen Theorien, die auch die Wirksamkeit des Völkerrechts annehmen. Übersichtshalber siehe S. Schieder/M. Spindler (Hrsg.), Theorien der Internationalen Beziehungen, Opladen 2010.

31 Etwa H. Morgenthau, Macht und Frieden, Gütersloh 1963, S. 69: Internationale Politik ist "ein Kampf um die Macht. Wo immer die letzten Ziele der internationalen Politik liegen mögen, das unmittelbare Ziel ist stets die Macht."

32 Vgl. H.J. Morgenthau, Politics among Nationals. The Struggle for Power and Piece, 6. Aufl. New York, 1985, S. 295. Die beschriebene Auffassung findet sich spieltheoretisch heute noch bei J.L. Goldsmith/E.A. Posner The Limits of International Law, Oxford: Oxford University Press 2005. Für eine Kritik, siehe A.van Aaken, To Do Away with International Law? Some Limits to 'The Limits of International Law', 17 European Journal of International Law 2006, S. 289. Nun differenzierter E.A. Posner/A.O. Sykes, Economic Foundations of International Law, Cambridge/Mass: Harvard University Press 2013.

33 Vgl. S. Oeter, Chancen und Defizite internationaler Verrechtlichung: Was das Recht jenseits des Nationalstaates leisten kann, in: M. Zürn/B. Zangl (Hrsg.), Verrechtlichung - Baustein für Global Governance?, Bonn 2004, S. 46. 
folgung des Völkerrechts werden in der Regel die Metatheorien Rationalismus (Rational-Choice Ansatz) und Konstruktivismus ${ }^{34}$ grob unterschieden. ${ }^{35}$ Dabei herrschte lange Zeit ein Streit der Orthodoxien. Inzwischen aber nähern sich die Theorien einander an, um so die Effektivität des Völkerrechts umfassend zu erklären. ${ }^{36}$ Hier kann nur ein Kurzüberblick gegeben werden, der nur so weit reichen soll, wie zum Verständnis der Mechanismen zur Effektuierung des Völkerrechts notwendig ist.

Der Rational Choice Ansatz ${ }^{37}$ ist eine von Politikwissenschaftler und Ökonomen ${ }^{38}$ verwendete Verhaltensannahme zur Erklärung des Verhaltens von Akteuren, seien es Individuen, Unternehmen, IGOs, NGOs oder Staaten. Ein Akteur ist dann rational, wenn er seinen erwarteten Nutzen unter Restriktionen maximiert (Erwartungsnutzentheorem) oder satisfiziert. ${ }^{39}$ Das Modell beruht damit auf zwei Komponenten, die trotz der verschiedenen Weiterentwicklungen insbesondere durch die Psychologie bzw. Verhaltensökonomik grundlegend unangetastet geblieben sind: (1) Akteure handeln gemäß ihren Präferenzen, die als stabil angenommen werden. Staatenpräferenzen müssen nicht notwendigerweise nur in (militärischer oder ökonomischer) Macht bestehen. Es kann auch eine Präferenz für den Erhalt der natürlichen Umwelt oder für die Korruptionsbekämpfung bestehen. (2) Sich ändernde Umweltbedingungen (auch Beschränkungen oder Restriktionen genannt). Beschränkungen sind dabei als Absteckung von Handlungsmöglichkeitsräumen gedacht, d.h. sie wirken nicht nur restriktiv, sondern können auch Handlungsmöglichkeiten eröffnen (etwa zur Erforschung des Weltraumes) und kanalisieren Verhalten durch positive und negative Anreize. Für die Erklärung und Prognose von Verhaltenswirkungen wird davon ausgegangen, dass sich eher die Restriktionen ändern als die Präferenzen der jewei-

34 Dieser Begriff ist zunächst einmal unabhängig vom wissenschaftstheoretischen oder philosophischen Begriff.

35 J. v. Stein, Engines (Fn. 10), S. 478 unterscheidet Instrumentalisten von Normativisten: während erstere extrinsische Kosten-Nutzen-Kalküle betonen, stehen als Befolgungsmechanismus der letzteren eher Identitäten, Ideen und Legitimität der Norm im Vordergrund.

36 Vgl. nur J. Fearon/A. Wendt, Rationalism v. Constructivism: A Skeptical View, in: W. Carlsnaes/T. Risse/B.A. Simmons (Hrsg.), Handbook (Fn. 10), S. 52; A. Thompson, Coercive Enforcement of International Law, in: J.L. Dunhoff/M.A. Pollack (Hrsg.), Perspectives (Fn. 1), S. 502 (508).

37 Ausführlich: A van Aaken, Rational-Choice in der Rechtswissenschaft. Zum Stellenwert der ökonomischen Theorie im Recht, Baden-Baden: Nomos 2003.

38 D. Snidal, Rational-Choice and International Relations, in: W. Carlsnaes/T. Risse/B.A. Simmons (Hrsg.), Handbook (Fn. 10), S. 73 sowie für die Ökonomen A. T. Guzman, How International Law Works (Fn. 10), sowie J.L. Dunoff/J.P. Trachtman, Economic Analysis of International Law, 24 Yale Journal of International Law 1999, S. 1; J.P. Trachtman, The Economic Structure of International Law, Cambridge, Mass.: Harvard University Press 2008. Für einen Überblick mit Literaturnachweisen siehe A.van Aaken, International Law: Rational Choice, in: (Hrsg.), Oxford Bibliographies Online, Oxford: Oxford University Press 2012, erhältlich unter: http://aboutobo.com/ (zuletzt besucht am 15. Mai 2013).

39 Nach Simon maximieren Individuen nicht, sondern satisfizieren nur, d.h. sie sind nur begrenzt rational und streben nur befriedigende, nicht maximale Lösungen an (das bedeutet, dass keine kontinuierliche Nutzenfunktion vorliegt, sondern eine Bewertung durch diskrete Anspruchsniveaus vorgenommen wird). H.A. Simon, Models of Man, London et al.: J. Wiley 1957. Für Staaten wird dies vertreten etwa von A. Chayes/A. Handler Chayes, Compliance (Fn. 12), S. 197 ff. 
ligen Akteure. Völkerrecht kann dadurch als Wirkungsfaktor analytisch isoliert werden, um brauchbare Erklärungen und auch Prognosen erstellen zu können. ${ }^{40}$

Innerhalb der Rational-Choice Theorien ist einerseits fraglich, welcher Stellenwert dem Völkerrecht (und soft law) für die Verhaltenswirkung eingeräumt wird und andererseits, ob der Staat als "black box" bzw. "billiard ball" ${ }^{41}$ gesehen wird oder ob ein Zwei-Ebenen-Spiel ${ }^{42}$ vorliegt (mit anderen Worten, ob die Wirkung des Völkerrechts im nationalen Recht bzw. in der nationalen Politik miteinbezogen wird). Letzteres ist auch aus rechtswissenschaftlicher Sicht zentral.

Das Spektrum der Rational-Choice Ansätze beginnt bei den klassischen Realisten, die dem Recht keinerlei Verhaltenswirkung zusprechen ${ }^{43}$ und reicht bis zu den (liberalen) Institutionalisten ${ }^{44}$ und Regimetheoretikern, ${ }^{45}$ in deren Theorien das Recht eine zentrale Rolle für das Verhalten von Staaten spielt. Realisten sehen schon deshalb keine Verhaltenswirksamkeit des Völkerrechts, weil die Durchsetzung des Rechts auf dem Handeln der anderen Staaten beruht, diese Durchsetzung aber ihrerseits kostspielig ist, weshalb Staaten in aller Regel davon absehen. Das Fehlen einer zentralen Durchsetzungsinstanz ist aber nicht das Ende der Geschichte. Andere Mechanismen werden diskutiert und insbesondere von Institutionalisten und Regimetheoretikern untersucht. Dennoch bleiben die zentralen Annahmen des Rational-Choice Ansatzes (die Betonung von Zwang, von Kosten-Nutzen-Analysen der relevanten Akteure und von materiellen oder anderen Anreizen, die u.a. durch das Recht gesetzt werden) erhalten. Auch die Analyse der Transaktionskosten, wie etwa

40 Dies ist problematisch bei konstruktivistischen Theorien, die eine Änderung der Präferenzen zulassen. Umweltbedingungen (formelle und informelle Institutionen; Restriktionen) als explanans und das Akteursverhalten als explanandum werden analytisch zusammengefasst. Motivation bzw. Präferenz und Restriktion fallen zusammen. Dadurch kann Recht als Wirkungsfaktor analytisch nicht isoliert werden. Aber auch bei den Konstruktivisten hat sich die Methode geändert, um so empirische Forschung und das Testen von Hypothesen zu erlauben, vgl. J.T. Checkel, Why Comply? Social Learning and European Identity Change, 55 International Organization 2001, S. 553 (565 ff.).

41 A.-M. Slaughter, International Law and International Relations: Millenial Lectures, Receuil de Cours/ Hague Academy of International Law 2001, S. 9 und zuletzt A.-M. Slaughter, International Law and International Relations Theory: Twenty Years Later, in: J.L. Dunhoff/M.A. Pollack (Hrsg.), Perspectives (Fn. 1), S. 613.

42 R.D. Putnam, Diplomacy (Fn. 6).

43 H.J. Morgenthau, Positivism, Functionalism, and International Law, 34 American Journal of International Law 1940, S. 260 sowie Ders., Politics among Nations. The Struggle for Power and Piece, 2. Aufl. New York: Knopf 1954, J. Mearsheimer, The Tragedy of Great Power Politics, New York: Norton, 2001.

44 Die Theorie der komplexen Interdependenz von Staaten ist dargestellt in R. Keohane/J. Nye, Power and Interdependence, Boston: Little Brown 1977 sowie R.O. Keohane, International Institutions and State Power: Essays in International Relations Theory, Boulder: Westview 1989. Zur liberalen Theorie: A. Moravcsik, Taking Preferences Seriously: A Liberal Theory of International Politics, 51 International Organization, 1997, S. 513.

45 V. Rittberger (Hrsg.), Regime Theory and International Relations, Oxford: Clarendon Press 1993 und B. Zangl, Interessen auf zwei Ebenen, Baden-Baden 1999. 
die Verminderung derselben zur Förderung internationaler Kooperation, fällt darunter. ${ }^{46}$

Diesen “interessengeleiteten” Ansätzen werden die sog. "normativen” Ansätze, die insbesondere auf konstruktivistischen Theorien beruhen, gegenübergestellt. ${ }^{47} \mathrm{Wie}$ auch die Rational-Choice Ansätze sind sie divers; geeint werden sie durch die Ansicht, dass jedenfalls nicht allein extrinsische Motivation die Staaten instrumental zur Befolgung anhält. Vielmehr seien Ideen und Normen wichtig, das „Management“ dieser Normen wie auch die Perzeption von Fairness und Legitimität des Rechts. Konstruktivisten (oder kritische Theoretiker) ${ }^{48}$ halten die Präferenzen der Akteure nicht für konstant, sondern betonen Lerneffekte, Ideologien, Kultur, gemeinsame Überzeugungen, Sozialisierung und soziale Normen wie auch argumentative Überzeugung. ${ }^{49}$ Alle diese Effekte können zur Befolgung des Völkerrechts beitragen und zwar sowohl auf einer rein zwischenstaatlichen Ebene als auch im innerstaatlichen Bereich. Ebenso sind psychologische Theorien zu finden, die von der Annahme des rationalen

46 Und daher auch Teile des "managerial approach" von A. Chayes/A. Handler Chayes, Compliance (Fn. 12).

47 J. v. Stein, Engines (Fn. 10), S. $485 \mathrm{ff}$.

48 Überblickshalber, siehe E. Adler, Constructivism and International Relations, in: W. Carlsnaes/T. Risse/B.A. Simmons (Hrsg.), Handbook (Fn. 10), S. 95. Ausführlich siehe A. Wendt, Constructing International Politics, 20 International Security 1995, S. 71 (71 f.): “Critical IR 'theory,' however, is not a single theory. It is a family of theories that includes postmodernists (Ashley, Walker), constructivists (Adler, Kratochwil, Ruggie, and now Katzenstein), neo-Marxists (Cox, Gill), feminists (Peterson, Sylvester), and others. What unites them is a concern with how world politics is 'socially constructed,' which involves two basic claims: that the fundamental structures of international politics are social rather than strictly material (a claim that opposes materialism), and that these structures shape actors' identities and interests, rather than just their behavior (a claim that opposes rationalism)".

49 Vgl. A. Wendt, Politics (Fn. 48), S. 73: "Social structures have three elements: shared knowledge, material resources, and practices. First, social structures are defined, in part, by shared understandings, expectations, or knowledge. These constitute the actors in a situation and the nature of their relationship, whether cooperative or conflictual. A security dilemma, for example, is a social structure composed of intersubjective understandings in which states are so distrustful that they make worstcase assumptions about each other's intentions, and as a result define their interests in self-help terms. A security community is a different social structure, one composed of shared knowledge in which states trust one another to resolve disputes without war. This dependence of social structure on ideas is the sense in which constructivism has an idealist (or 'idea-ist') view of structure. What makes these ideas (and thus structure) 'social,' however, is their intersubjective quality. In other words, sociality (in contrast to 'materiality,' in the sense of brute physical capabilities), is about shared knowledge." Vgl. auch M. Finnemore, National Interests in International Society, Ithaca/London: Cornell University Press 1996, S. 128: "The fact that we live in an international society means that what we want and, in some ways, who we are are shaped by the social norms, rules, understandings, and relationships we have with others. Theses social realities are as influential as material realities in determining behaviour. Indeed, they are what endow material realities with meaning and purpose. In political terms, it is these social realities that provide us with ends to which power and wealth can be used.” 
Handelns der Staaten abweichen. ${ }^{50}$ Hier ergeben sich spannende Forschungsfelder im Bereich der Verhaltensökonomie und der "behavioral game theory". 51

In der Regel sind die Ansätze optimistisch in dem Sinne, dass es eine "propensity to comply” gibt. Da Staaten freiwillig Verträge schließen (Konsensprinzip des Völkerrechts), ist anzunehmen, dass sie diese auch einhalten wollen. ${ }^{52}$ Tun sie es nicht, so wird dies auf drei Ursachen zurückgeführt: 1) Die Befolgung ist nicht eindeutig definiert; Verträge sind unvollständig und manche Verhaltensweisen werden nicht ex ante bedacht. ${ }^{53}$ Es kommt dann maßgeblich auf die Flexibilitätsmechanismen an, die der Vertrag anbietet bzw. darauf, ob es eine bindende Auslegungsinstanz gibt. ${ }^{54}$ 2) Weiterhin fehlt es Staaten oft an der notwendigen Kapazität, ${ }^{55}$ um Völkerrecht zu befolgen. Beispiele reichen von Transparenzanforderungen, welche die WTO an Staaten stellt, ${ }^{56}$ bis hin zu überlangen Gerichtsverfahren oder überfüllten Gefängnissen. 3) Die zeitliche Dimension kann auch dazu beitragen, dass Staaten Anpassungs- und Übergangsfristen brauchen, um eine Vertragserfüllung garantieren zu können. ${ }^{57}$

Um eine größere Befolgung sicherzustellen, würden “managerialists” bei Transparenz, Rechtsklarheit, Streitschlichtungsmechanismen sowie technischer und finanzieller Hilfe ansetzen. ${ }^{58}$ Auch aus rechtswissenschaftlicher Sicht wurde die Präzision von Verträgen als Legitimationsmechanismus betrachtet, der die Kohärenz des Rechts fördert und einen "compliance pull" auslöst. ${ }^{59}$ Auch Normen und Identität werden als maßgeblich für die Befolgung des Völkerrechts angesehen; die Internalisierung von Normen gilt als ein entscheidendes Kriterium. Dies kann sowohl über

50 J. Gross Stein, Psychological Explanantions of International Conflict, in: W. Carlsnaes/T. Risse/B.A. Simmons (Hrsg.), Handbook (Fn. 10), S. 292. Zu psychologischen Ansätzen in der Theorie der Internationalen Beziehungen R. McDermott, Political Psychology in International Relations, Ann Arbor: University of Michigan Press 2004.

51 C. Camerer, Behavioral Game Theory. Experiments in Strategic Interaction, Princeton: Princeton University Press 2003; A. van Aaken, Towards Behavioral International Law and Economics? Comment on Kenneth W. Abbott, 2008 Illinois Law Review 2008, S. 47 sowie die Nachweise in (Fn. 63).

52 A. Chayes/A. Handler Chayes, Compliance (Fn. 12), S. 186.

53 Diese Auffassung ist auch (ökonomisch) vertragstheoretisch reformuliert, vgl. R.E. Scott/P.B. Stephan, Leviathan (Fn. 27).

54 Vgl. die Nachweise in (Fn. 27).

55 A. Chayes/A. Handler Chayes, Compliance (Fn. 12), S. $193 \mathrm{ff.}$

56 P. Ala'i, From the Periphery to the Center? The Evolving WTO Jurisprudence on Transparency and Good Governance, 11 Jounal of International Economic Law 2008, S. 779.

57 A. Chayes/A. Handler Chayes, Compliance (Fn. 12), S. 195 ff.: "time lag between undertaking and performance".

58 Vgl. auch P.M. Haas, Do Regimes Matter? Epistemic Communities and Mediterranean Pollution Control, 43 International Organization 1998, S. 377 in Bezug auf technische Expertise.

59 T.M. Franck, The Power of Legitimacy among Nations, New York: Oxford University Press 1990. 
transnationale Beeinflussungs- und Überzeugungsprozesse geschehen, ${ }^{60}$ als auch durch nationale Prozesse. ${ }^{61}$

Auch Fairness und Legitimität des Völkerrechts werden als Faktoren der Normbefolgung genannt. Regeln werden dann anerkannt, wenn sie durch einen fairen Prozess entstanden sind und in einem solchen angewendet werden. ${ }^{62}$ Rechtsklarheit und Rechtsgleichheit sind dabei wichtige Komponenten. Hier schließt sich wiederum der Kreis mit den verhaltensökonomischen Theorien, ${ }^{63}$ die ihrerseits systematische Abweichungen vom Rationalverhalten untersuchen und auch Fairnesspräferenzen sowie die Bedeutung von Verfahren analysieren. ${ }^{64}$ Im Folgenden werden diese Ansätze kombiniert, um so die mannigfaltigen Wirkungen von Völkerrecht (und soft law) aufzeigen zu können. Ausgangspunkt der Analyse bleibt stets die Rational-Choice Theorie.

\section{Reziprozität, Reputation und Retaliation: die Problemfelder}

Retaliation, Reziprozität und Reputation werden als die Wirkmechanismen betrachtet, durch die Völkerrecht, trotz fehlender Durchsetzungsinstanz, effektuiert wird. ${ }^{65}$ Es gibt Völkerrecht, welches sehr gut befolgt wird, und solches, bei dem weithin anerkannt wird, dass Durchsetzungsschwierigkeiten bestehen. Geht man auf die zugrundeliegenden Problemstrukturen unter Rückgriff auf spieltheoretische Konzepte zurück (also basierend auf der Annahme des strategischen Verhaltens), so bietet

60 H.H. Koh, Why do Nations Obey International Law?, 106 Yale Law Journal 1997, S. 2599.

61 T. Risse/K. Sikkink, The Socialization of International Human Rights Norms into Domestic Practices: Introduction, in: T. Risse/S.C. Ropp/K. Sikkink (Hrsg.), The Power of Human Rights: International Norms and Domestic Change, Cambridge: Cambridge University Press 1999, S. 1 sowie M. Finnemore/K. Sikkink, International Norm Dynamics and Political Change, 52 International Organization 1998, S. 887.

62 T.M. Franck, Legitimacy (Fn. 59). Für das nationale Recht, siehe grundlegend T.R. Tyler, Why People Obey the Law, New Haven et al.: Yale University Press 1990; T.R. Tyler, A Psychological Perspective on the Legitimacy of Institutions and Authorities, in: J.T. Jost/B. Major (Hrsg.), The Psychology of Legitimacy, Cambridge: Cambridge University Press 2001, S. 416.

63 Zur verhaltensökonomischen Theorie des (nationalen) Rechts siehe statt vieler C. Engel/M. Englerth/ J. Lüdemann/I. Spiecker gen. Döhmann (Hrsg.), Recht und Verhalten. Beiträge zu Behavioral Law and Economics, Tübingen 2007; C. Jolls/C.R. Sunstein/R.H. Thaler, A Behavioral Approach to Law and Economics, 50 Stanford Law Review 1998, S. 1471; C.R. Sunstein (Hrsg.), Behavioral Law and Economics, Cambridge: Cambridge University Press 2000. Zur Anwendung auf das Völkerrecht siehe A.van Aaken, Comment (Fn. 51), S. 47, L.N. Poulsen/E. Aisbett, When the Claim Hits: Bilateral Investment Treaties and Bounded Rational Learning, 65 World Politics, 2013, S. 273 sowie J. Galbraith, Treaty Options: Towards a Behavioral Understanding of Treaty Design, 53 Virginia Journal of International Law, 2013, S. 309. Ausführlich zum Potential dieser Forschung auch im Hinblick auf Effektuierung A. van Aaken, Behavioral International Law and Economics, Working Paper, erhältlich auf SSRN.

64 A. van Aaken, Das deliberative Element juristischer Verfahren als Instrument zur Überwindung nachteiliger Verhaltensanomalien. Ein Plädoyer für die Einbeziehung diskursiver Elemente in die Verhaltensökonomik des Rechts, in: C. Engel/M. Englerth/J. Lüdemann/I. Spiecker gen. Döhmann (Hrsg.), Verhalten (Fn. 63), S. 189.

65 A. T. Guzman, How International Law Works (Fn. 10). Er spricht von den "Three 'Rs' of Compliance": Retaliation, Reputation, Reciprocity. 
es sich an, zwischen Koordinationsspielen - etwa der Festlegung einer einheitlichen Sprache in der Flugsicherung - und Kooperationsspielen zu unterscheiden. ${ }^{66}$ Bei Koordinationsspielen hat kein Staat, sobald die Norm einmal festgelegt ist, ein Interesse daran, die Norm zu verletzen, da abweichendes Verhalten selbstschädigend wäre (ähnlich wie bei Falschfahrern). Völkerrecht besitzt hier die Funktion, Verhalten mittels eines Fokalpunktes zu koordinieren; einen Standard festzulegen, der ohne weiteres von allen Staaten eingehalten wird. Regelgeltungs- und Regelbefolgungsinteresse fallen hier zusammen: ein Effektuierungsproblem ergibt sich nicht.

In allen übrigen Fällen der Kooperationsspiele kann das Regelbefolgungsinteresse zu einem bestimmten Zeitpunkt nicht gegeben sein, sei es aufgrund mangelnden Vertrauens in das Verhalten anderer Staaten (stag hunt game), ${ }^{67}$ sei es, weil ein Staat sich immer besser stellt, wenn er sich nicht an die Norm hält (ein Gefangenendilemma). ${ }^{68}$ Das Gefangendilemma war das Standardspiel, welches etwa von Realisten in Sicherheits- und Abrüstungsfragen verwendet wurde. Es kommt auch in allen Fällen zum Tragen, in denen Staaten kooperieren, um ein öffentliches Gut herzustellen oder zu erhalten, ${ }^{69}$ etwa im Umweltschutz oder im Menschenrechtsschutz: ${ }^{70}$ Wird dieselbe Situation als Vertrauensspiel dargestellt, so ergeben sich andere Anreize für die Akteure und damit andere Verhaltensprognosen und ggf. institutionelle Folgen, etwa in Bezug auf Durchsetzungsmechanismen oder Transparenz- und Monitoringvorschriften. Ebenso öffnet sich dann die Tür für konstruktivistische Ansätze, welche die internen (Lern-)Prozesse vermehrt betonen.

$\mathrm{Zu}$ den Wirkmechanismen im Einzelnen: Retaliation betrifft Fälle, in denen direkte Sanktionsmechanismen angewendet werden, also Zwang gegen den völkerrechtsverletzenden Staat. ${ }^{71}$ Die Befolgung von Völkerrecht zu erzwingen wirft das Problem auf, dass andere Staaten bereit sein müssen, Völkerrechtsbrüche zu sanktionieren, denn Rechtsdurchsetzung ist kostspielig. Die Anreize für Staaten, Rechtsdurchsetzung bereitzustellen (Retaliation), ist weniger gut erforscht als die Frage, warum sich Staaten an Völkerrecht halten. Die einfachste Antwort ist, dass Rechtsdurchsetzung nur dann zu erwarten ist, wenn der Nutzen daraus, den Rechtsbruch zu sanktionie-

66 Im Detail zur Spieltheorie in Normkontexten, siehe D.G. Baird/R. Gertner/R.C. Picker, Game Theory and the Law, Cambridge (Mass.): Harvard University Press 1994; J.D. Morrow, Game Theory for Political Scientists, New Jersey: Princeton University Press 1994.

67 B. Skyrms, The Stag Hunt and the Evolution of Social Structure, Cambridge: Cambridge University Press 2004.

$68 \mathrm{Zu}$ einer Kritik der jetzigen spieltheoretischen Verwendung im Recht J.D. Oblin, Nash Equilibrium and International Law, 23 European Journal of International Law 2012, S. 915, der für die Anerkennung der Normativität des Rechts als Handlungsgrund plädiert.

69 Vgl. zu den globalen öffentlichen Gütern nun F. Cafaggi/D.D. Caron, Global Public Goods amidst a Plurality of Legal Orders: A Symposium, 23 European Journal of International Law 2012, S. 643.

70 Dieser kann als normatives öffentlichen Gut betrachtet werden; juristisch betrachtet sind es inter omnes Verpflichtungen.

71 A. T. Guzman, How International Law Works (Fn. 10). 
ren, die Kosten des Sanktionierens übersteigt. Steht zudem ein Kollektivgut in Frage, dann besteht für jeden Staat ein Anreiz, darauf zu warten, dass ein anderer Staat die Kosten der Sanktion auf sich nimmt (Trittbrettfahrerproblem oder "sanctioners' dilemma”), ${ }^{72}$ denn ein Staat, der andere Völkerrechtsverletzer sanktioniert, stellt ein öffentliches Gut bereit. Wie Snidal schreibt: “This outcome will be most likely when some single state, the hegemonic power, is sufficiently large relative to the others so that it will capture a share of the benefit of the public good larger than the entire cost of providing it." 73 Der Nutzen des sanktionierenden Staates muss sich aber nicht auf die Wiederherstellung des rechtmäßigen Zustandes durch den verletzenden Staat beschränken; er kann auch darin bestehen, dass generalpräventiv dritte Staaten von einem Völkerrechtsbruch abgehalten werden. ${ }^{74}$ Hierzu muss für den sanktionierenden Staat oder das Kollektiv der sanktionierenden Staaten das Rechtsgeltungsinteresse hoch sein.

Im besten Falle sind Verträge oder soft law Normen selbstdurchsetzend. Ein selbstdurchsetzendes Abkommen ist ein solches, an das sich zwei oder mehrere Parteien halten, so lange als der Nutzen aus dem Bestand des Abkommens den Nutzen der Aufhebung des Abkommens übersteigt (Regelgeltungsinteresse). ${ }^{75}$ Dies bedeutet nicht, dass diese Normen keiner Sanktionen bedürfen, vielmehr fließen die Sanktionen aus dem Verhalten der Vertragsparteien. Sie sind daher nicht auf die Durchsetzung durch externe Mechanismen oder Drittparteien angewiesen. Es liegt in der $\mathrm{Na}$ tur der Abkommen selbst, dass hinreichend Anreize für ihre Befolgung bestehen. Der erwartete Langzeitnutzen des Abkommens übersteigt den gegenwärtigen Wert einer Verletzung des Abkommens. Die Norm wird mithin dadurch durchgesetzt, dass der zukünftige Nutzenstrom für den verletzenden Staat aus dem Abkommen ausgeschlossen oder reduziert wird.

Der bekannteste und klassische Mechanismus hierfür ist die Reziprozität. Dies spiegelt sich insbesondere im Recht, etwa in Art. 60 der Wiener Vertragsrechtskonvention, welcher die Beendigung oder Suspendierung eines Vertrags infolge erheblicher Vertragsverletzung (material breach) vorsieht. Reziproke Verträge, insbesondere bilaterale Verträge, sind daher inhärent selbstdurchsetzend. Reziprozität ist von Spieltheoretikern wie auch Rechtswissenschaftlern als "engine of cooperation" 76 bezeich-

72 A. Thompson, Coercive Enforcement (Fn. 36), S. 511. Spieltheoretisch ausgedrückt stellt sich hier ein "second-order enforcement problem", welches als Gefangenendilemma dargestellt werden kann, da die Durchsetzung des Rechts ein öffentlichen Gut ist. Auch wenn alle Staaten sich besser stellen würden, wenn das Völkerrecht durchgesetzt würde, ist ein Trittbrettfahrerverhalten individuell rational.

73 D. Snidal, The Limits of Hegemonic Stability Theory, 39 International Organization 1985, S. 579 (581).

74 A. Thompson, Coercive Enforcement (Fn. 36), S. 503.

75 Definition in Anlehnung an B. Simmons, Mobilizing (Fn. 8), S. 116.

76 J.v. Stein, Engines (Fn. 10), S. 480. 
net worden. ${ }^{77}$ Bereits die Drohung, auf einen Rechtsbruch mit einem "Rechtsbruch" zu antworten, kann den ersten Rechtsbruch verhindern, vorausgesetzt, der Schatten der Zukunft ist groß genug (d.h. die Kosten der Vertragsbeendigung, Rechtsgeltungsinteresse). Wichtigste Bedingung für die Effektuierung des Völkerrechts durch Reziprozität ist, dass reziproke Nichtbefolgung demjenigen Staat Kosten auferlegt, welcher den Rechtsbruch begehen will. Weiterhin muss eruiert werden, ob ein Rechtsbruch tatsächlich stattgefunden hat oder nicht. Schwierig ist dies insbesondere dann, wenn Rechtsadressaten letztlich Individuen oder Firmen statt Staaten sind.

Nicht-rezipoke Verträge, wie etwa Menschenrechtsverträge oder humanitäres Recht, ${ }^{78}$ können nicht auf eine solche Art der Selbstdurchsetzung bauen; sie bedürfen anderer Durchsetzungsmechanismen: ein Staat kann nicht die eigenen Menschenrechtsverpflichtungen suspendieren, weil ein anderer Staat Menschenrechte erheblich verletzt. Allerdings können diese Verträge mit anderen, reziproken Abkommen auf eine Weise verbunden werden, dass Reziprozität spielt (Linkages).

Ein weiterer Wirkmechanismus zur Selbstdurchsetzung des Völkerrechts ist die Reputation der Staaten. ${ }^{79}$ Wird die Reputation eines Staates als verlässlicher Partner aufgrund von Völkerrechtsverletzungen in Mitleidenschaft gezogen, so kann dies andere Staaten dazu bewegen, zukünftige Kooperationen mit diesem Staat nicht mehr einzugehen. Spieltheoretisch betrachtet erhöht dieses „Drohpotential“ die Kosten der Defektion (also der Nichteinhaltung der Norm), sofern der Staat die Kooperationsverweigerung antizipiert. Dies gilt jedenfalls bei wiederholten Spielen. ${ }^{80}$ Wenn der Nutzen aus zukünftiger Kooperation mit anderen Staaten groß genug ist ${ }^{81}$ und die Wahrscheinlichkeit der Sanktion durch Nichtkooperation ausreichend hoch, dann werden sich Staaten selbst bei Fehlen eines kurzfristigen Regelbefolgungsinteresses an die jeweilige Norm halten. Reputationsverlust kann sich sowohl aus dem Verstoß gegen Völkervertragsrecht als auch Völkergewohnheitsrecht ergeben. Der Reputationsverlust ist umso stärker, je gravierender der Normverstoß bzw. umso wichtiger die Norm ist (etwa ius cogens). Strittig ist allerdings, ob Reputation rechts-

77 R. Axelrod, The Evolution of Cooperation, New York: Basic Books 1984, B. Simma, Zustandekommen (Fn. 5); O. Schachter, International Law in Theory and Practice, Dordrecht: Martinus Nijhoff Press 1991; J.v. Stein, Engines (Fn. 10), S. 480.

78 Art. 60 Abs. 5 WVRK.

79 Früh R. Keohane, After Hegemony, Princeton: Princeton University Press 1984, S. 106, der einen Grund der Befolgung des Völkerrechts im Interesse der Staaten an weiterer, zukünftiger Kooperation erblickt, auch wenn es in ihrem kurzfristige Interesse läge, das Völkerrecht zu brechen. Er nennt "reasons of reputation, as well as fear of retaliation and concern about the effects of precedents." Umfassend jetzt R. Brewster, Reputation in International Relations and International Law, in: J.L. Dunhoff/ M.A. Pollack (Hrsg.), Perspectives (Fn. 1), S. 524.

80 Da Staaten in aller Regel nicht untergehen, sind die Spiele im Völkerrecht wiederholt.

81 Ist der Zeithorizont der Kooperation lang genug, dann ist der Diskontfaktor niedrig. 
gebietsspezifisch ${ }^{82}$ oder umfassend ${ }^{83}$ ist. $^{84}$ Im ersteren Falle wäre die Effektuierungswirkung erheblich schwächer. Ein Staat könnte dann etwa Umweltvölkerrecht missachten, ohne dadurch Reputation und Kooperationsmöglichkeiten im Handelsrecht einzubüßen. In gleicher Weise würden sich Menschenrechtsverletzungen nicht auf die Kooperationsbereitschaft im Sicherheitsbereich auswirken. Weiterhin bezieht sich ggf. ein Reputationsverlust nur auf die Regierung und zieht nicht zwangsläufig die Reputation eines ganzen Landes für einen langen Zeitraum in Mitleidenschaft. ${ }^{85}$

Der Reputationsmechanismus stößt an Grenzen zwischen Staaten, die nicht viel miteinander interagieren, wenn es sich um Staaten handelt, die kein großes Interesse an internationaler Kooperation haben sowie im Hinblick auf Staaten, die daran interessiert sind, als "starker" Staat wahrgenommen zu werden, der seine Interessen oder Verbündeten ungeachtet einer möglichen Völkerrechtsverletzung schützt. ${ }^{86} \mathrm{Er}$ setzt überdies voraus, dass Verstöße beobachtet werden können und sich ein Verhalten als Verstoß qualifizieren lässt. Hier kommen Monitoringmechanismen, Berichtspflichten sowie (internationale) Gerichte zum Tragen. Insoweit ist es für die Reputation unerheblich, ob es sich um Urteile oder Gutachten der Gerichte handelt bzw. ob es unabhängige Gremien gibt, die - wie etwa das Menschenrechtskomitee des Internationalen Paktes über politische und bürgerliche Rechte (IPBürg) - nur Auffassungen (views) generieren. Maßgeblich für die Frage der Reputationseffekte ist allein eine legitimierte autoritative Entscheidungsinstanz.

Auch nichtstaatliche Akteure wie NGOs spielen eine ganz erhebliche Rolle in der Aufdeckung und Bekanntmachung von Völkerrechtsverstößen, etwa im Menschenrechts- oder Umweltbereich. Ein gerichtlich festgestellter Verstoß kann erheblich höhere Reputationsrisiken bergen als die bloße Einschätzung der beteiligten Staaten, welche allen Parteien eigeninteressierte Auslegungen erlaubt.

Reputationseffekte allein aber sind nicht hinreichend, wenn es keine kollektive Antwort im Sinne einer zukünftigen Kooperationsverweigerung gibt. Wiederum sehen sich Staaten hier mit einem Kollektivhandelsproblem konfrontiert. ${ }^{87}$ Fehlt daher ein zentraler Durchsetzungsmechanismus, so muss der Vertrag selbstdurchsetzend sein. Bei allen übrigen Verträgen wird aus Rational-Choice-Sicht keine Befolgung prognostiziert, es sei denn, es existieren zusätzliche innerstaatliche Mechanismen. Die

82 So G.W. Downs/M.A. Jones, Reputation, Compliance, and International Law, 31 Journal of Legal Studies 2002, S. 95.

83 So Guzman, How International Law Works (Fn. 10), S. $71 \mathrm{ff}$.

84 Vgl. auch $v$. Stein, Engines (Fn. 10), S. 481 f. sowie zu einer verhaltensökonomischen Beurteilung van Aaken, Behavioral International Law and Economics (Fn. 63).

85 Brewster, Reputation (Fn. 79), S. 534 f. sowie Posner/Sykes, Economic Foundations (Fn. ), S. 33.

86 R.O. Keohane, International Relations and International Law: Two Optics, 38 Harvard International Law Journal 1997, S. 487.

87 Guzman, Compliance (Fn. 10). 
empirischen Studien zum Reputationsmechanismus zeigen gemischte Resultate, die zudem eher auf einen kompartimentalisierten Reputationseffekt hindeuten. ${ }^{88}$

Dennoch gibt es eine Rational-Choice Logik, die erklären kann, warum Verträge geschlossen werden: sie sind - mehr noch als soft law oder Exekutivabkommen ein "commitment device", 89 welches Staaten erlaubt, glaubhafte Versprechen zu machen. Dies gilt umso mehr, je schwieriger das nationale Ratifikationsverfahren ist und je "härter" die Verträge sind (in dem Sinne, dass sie wenig normative Flexibilität einräumen, also tendenziell bestimmte Rechtsbegriffe beinhalten) und je mehr Effektuierungsmechanismen sie enthalten (etwa obligatorische Streitschlichtung). ${ }^{90}$

\section{Klassische Mechanismen zur Effektuierung des Völkerrechts}

Aus rechtswissenschaftlicher Sicht gilt das Primat des Rechts über Politik bzw. Macht. Auch wenn die "Machtsensibilität" 91 des Völkerrechts nicht geleugnet werden kann, so wird diese heutzutage doch zumindest rechtlich eingehegt. ${ }^{92}$ Streitigkeiten und Nichtbefolgung von Recht sind primär friedlich beizulegen; "gun-boat diplomacy" ist nicht mehr erlaubt. Die Völkerrechtswissenschaft hat sich zur Effektuierung des Völkerrechts zumeist auf Streitbeilegungsmechanismen und (legale) Zwangsmaßnahmen sowie auf innerstaatliche Mechanismen verlassen.

\section{Zentrale Durchsetzung des Völkerrechts}

Zwar wird das Völkerrecht oft als dezentrale Rechtsordnung bezeichnet, doch existieren auch zentrale Durchsetzungsmechanismen. ${ }^{93}$ Als solche werden hier Mechanismen verstanden, in denen Staaten kollektiv auf Völkerrechtsbrüche reagieren bzw. in denen sie kollektiv, ggf. durch IGOs, handeln. Wichtigstes Beispiel ist das System der kollektiven Sicherheit im Rahmen der Vereinten Nationen. ${ }^{94}$ Nur in den Ausnahmefällen der Selbstverteidigung oder eines Sicherheitsratsbeschlusses darf ein Rechtsbruch mit Gewalt sanktioniert werden. Zwang als Mittel zur Durchsetzung des Völkerrechts effektuiert das Völkerrecht nach klassischen Rechtstheorien wie auch den realistischen Theorien der Internationalen Beziehungen. Der Sicherheitsrat

88 Allerdings ist die Forschung noch relativ neu. Übersichtshalber, siehe Brewster, Reputation (Fn. 79), S. $530 \mathrm{f}$.

89 Simmons, Mobilizing (Fn. 8), S. $118 \mathrm{ff}$.

90 G.C. Shaffer/M.A. Pollack, Hard Law and Soft Law, in: J.L. Dunhoff/M.A. Pollack (Hrsg.), Perspectives (Fn. 1), S. 203 erwähnen mehrere Mechanismen, warum "hard law" in der Durchsetzung Vorteile gegenüber soft law genießt. Erstens hat hard law einen höheren Reputationswert und zieht rechtliche Sanktionen nach sich. Zweitens hat hard law u.U. einen direkten Effekt in nationalen Rechtsordnungen, welcher nationale Akteure zur Durchsetzung des Rechts bewegen können. Drittens enthalten hard law-Normen oft die Delegation der Interpretation an Drittparteien und lösen so das Problem unvollständiger Verträge. Viertens erlauben sie die Überwachung und ggf. die Feststellung des Verstosses.

91 Vöneky, Durchsetzung (Fn. 3), S. 490.

92 Vgl. Oeter, Chancen und Defizite (Fn. 33).

93 J.A. Frowein, Collective Enforcement of International Obligations, 47 ZaöRV 1987, S. 67.

94 Gemäß Kapitel VII der Charter der Vereinten Nationen. 
kann sowohl friedliche als auch militärische Maßnahmen anordnen und besitzt hierbei weites Ermessen; die durch ihn bestimmten Maßnahmen allerdings stehen unter dem Vorbehalt der Politik. Durch die fünf Vetomächte kommt es zudem zu einer selektiven Durchsetzung des Völkerrechts. Weiterhin sind die Beschlüsse nicht durch den IGH überprüfbar, was ihre Legitimität einschränken kann. ${ }^{95}$

$\mathrm{Zu}$ betonen bleibt, dass - abgesehen von der Blockade des Sicherheitsrates während des kalten Krieges - nach wie vor erklärt werden muss, weshalb Staaten Kosten aufwenden, um kollektiv Recht durchzusetzen: das o.g. "sanctioner's dilemma” kommt hier zum Tragen. Neben den materiellen Kosten ${ }^{96}$ entstehen für den Sanktionierer auch politische Kosten, sowohl auf nationaler Ebene als auch auf internationaler Ebene. Auf nationaler Ebene ist es z.B. häufig unpopulär, Truppen in internationale Einsätze zu entsenden; auf internationaler Ebene fällt es schwer, andere Staaten davon zu überzeugen, dass nicht nur aus Eigeninteresse gehandelt wird. Eine eindeutige Rechts- und Faktenlage kann in diesem Zusammenhang hilfreich sein; entpolitisierte Entscheidungsgremien spielen insofern auch hier eine wesentliche Rolle. Das Risiko, sich eine Reputation als Trittbrettfahrer einzuholen, mag Staaten ebenfalls zu einem Engagement bewegen.

Auch kollektive Wirtschaftssanktionen legen sowohl den Sanktionierern als auch dem sanktionierten Staaten Kosten auf (etwa Rohstoffknappheit oder Einbußen der exportierenden Industrie). Der Anreiz, die Sanktionen zu brechen, besteht also auch dort. Gemildert wird dieser Umstand allerdings durch den Einsatz von "smart sanctions". ${ }^{97}$ Im sanktionierten Staat werden die Verantwortlichen direkt sanktioniert; die Gesamtbevölkerung wird eher geschont. Auch für die Sanktionierenden indes sind „smart sanctions“ vorteilhaft: Der Reziprozitätsmechanismus entfällt, die Kosten sind geringer. Ob die Sanktionsaktivität von Staaten im Zeitalter der “smart sanctions" zugenommen hat, müsste empirisch untersucht werden.

95 Vgl. U.N. Doc. 664, IV/2/33 13 U.N.C.I.O. Docs 633 (1945) sowie Legal Consequences for States of the Continued Presence of South Africa in Namibia (South West Africa) Nonwithstanding SC Resolution 276 (1970), Gutachten vom 21. Juni 1971 ICJ Reports 45.

96 Regelmäßig ist es schwierig, Truppen und Geld dafür zusammen zu bekommen, obwohl die Finanzierung obligatorisch ist, vgl. Art. 17 UN Charter und VN-GA Res. A/RES/55/235 v. 23. Dezember 2000. Laut der UN Website on Peace-Keeping <https://www.un.org/en/peacekeeping/operations/financing.shtml> (zuletzt besucht am 15. Mai 2013): "Although the payment of peacekeeping assessments is mandatory, as of 28 February 2013, Member States owed approximately $\$ 2.98$ billion in current and back peacekeeping dues."

$97 \mathrm{Zu}$ den Argumenten Für und Wider siehe J. Gordon, Smart Sanctions Revisited, 25 Ethics \& International Affairs 2011, S. 315 sowie G.A. Lopez, In Defense of Smart Sanctions, 26 Ethics \& International Affairs 2012, S. 135. Zu Legitimitätsfragen siehe T. Stein, Too "smart" for legal protection? UN Security Council's targeted sanctions and a pladoyer for another UN tribunal, in: H. Hestermeyer/ D. König/N. Matz-Lück/V. Röben/A. Seibert-Fohr/P.-T. Stoll/S. Vöneky (Hrsg.), Coexistence, Cooperation and Solidarity. Liber Amicorum Rüdiger Wolfrum, Leiden: Martinus Nijhoff 2012, S. 1527. 
Aber nicht nur der Sicherheitsrat effektuiert das Völkerrecht, andere IGOs sind hierin ebenfalls aktiv. ${ }^{98}$ Bekannt sind etwa die Bretton Woods Institutionen, die mittels Konditionalitäten Umweltschutz- und Menschenrechtsnormen einbeziehen. ${ }^{99}$ Eine besondere Rolle kommt IGOs im Bereich der Informationssammlung, der Überwachung und des Monitoring zu. Dies gilt etwa für den Bereich der Menschenrechte, in denen die jeweiligen Komitees der Menschenrechtsverträge der Vereinten Nationen die Menschenrechtslage überwachen. Nicht zuletzt sind sie für den internationalen Umweltschutz zentral. ${ }^{100}$

\section{Dezentrale Durchsetzung auf internationaler Ebene}

Die dezentrale Durchsetzung des Völkerrechts steht im Mittelpunkt der Frage der Effektuierung des Völkerrechts. Hierbei stellen sich wiederum die o.g. Problemstrukturen der Reputation, der Retaliation und der Reziprozität. Die Durchsetzung des Völkerrechts ist aber lange nicht mehr auf die rein horizontale, zwischenstaatliche Ebene beschränkt (1.), vielmehr erfolgt die Durchsetzung auch vertikal, nämlich in der innerstaatlichen Ordnung (2.).

\section{Internationale Streitbeilegungsmechanismen}

Internationale Streitbeilegungsmechanismen erfolgen zwischenstaatlich oder über internationale Mechanismen, die von nichtstaatlichen Akteuren genutzt werden. Sie können zweierlei Form annehmen; nicht-gerichtsförmige oder gerichtliche Verfahren.

\section{a) Außergerichtliche Streitbeilegungsmechanismen}

$\mathrm{Zu}$ den außergerichtlichen Streitbeilegungsmechanismen gehören insbesondere die diplomatischen Verfahren, d.h. Verhandlung, Vermittlung, Untersuchung und Schlichtung. ${ }^{101}$ Solche Mechanismen effektuieren das Völkerrecht primär im Sinne der konstruktivistischen Ansätze. Sie versuchen, faire und für alle Beteiligten akzeptable Ergebnisse zu finden. Nach Erkenntnissen aus dem nationalen Recht haben ausgehandelte Ergebnisse bessere Befolgungschancen als gerichtlich auferlegte, ${ }^{102} \mathrm{da}$ sie ohne Zwang zu Stande kommen und als fair wahrgenommen werden. Ein Mehr an empirischer Forschung zur Effektuierung in diesem Bereich wäre wünschenswert.

98 E. Klein, Sanctions by International Organizations and Economic Communities, 30 AVR 1992, S. 3.

99 M. Herdegen, Der Beitrag des modernen Völkerrechts zu Good Governance, in: Rudolf Dolzer u.a. (Hrsg.), Good Governance: Gute Regierungsführung im 21. Jahrhundert, 2007, S. 107.

100 Vgl. A.E. Boyle, Saving the World - Implementation and Enforcement of International Environmental Law through International Institutions, 3 Journal of Environmental Law 1991, S. 229.

101 Vgl. Art. 33 UN Charter und C.C. Joyner, Reconciliation as Conflict Resolution, 8 New Zealand Journal of Public and International Law 2010, S. 39.

102 Vgl. R. Schmalz-Bruns, Deliberativer Supranationalismus, 2 Zeitschrift für Internationale Beziehungen 1999, S. 185; A.van Aaken, Deliberative Institutional Economics, or Does Homo Oeconomicus argue?, in: A.van Aaken/C. List/C. Lütge (Hrsg.), Deliberation and Decision. Economics, Constitutional Theory and Deliberative Democracy, Aldershot: Ashgate 2004, S. 3 m.w.N. 


\section{b) Zwischenstaatliche gerichtliche Verfahren}

Im Mittelpunkt der Diskussion zur Effektuierung des Völkerrechts steht neben den Sanktionen die gerichtliche Streitbelegung. ${ }^{103}$ Bekannte Beispiele internationaler Gerichte sind der IGH, der Appellate Body der WTO oder der Europäische Gerichtshof. Sie dienen als Alarm- und Informationsclearingsysteme. Dennoch bleibt zu beachten, dass Gerichte zwar gegen die Normverletzer entscheiden können, aber keine Möglichkeit haben, die Befolgung ihrer Urteile sicherzustellen. Hier kommt wiederum die Reziprozität zum Tragen: wird ein Normverstoß festgestellt, ermächtigt dies die anderen Staaten, Repressionen oder (falls das jeweilige Regime es vorsieht) andere Sanktionen zu ergreifen, die ihrerseits das System selbstdurchsetzend machen können, wie etwa in der WTO. Jedoch wird aus sozialwissenschaftlicher Sicht davon ausgegangen, dass die Gerichte politisch beschränkt sind: Urteile, die bereits ex ante keine Befolgung erwarten lassen, werden erst gar nicht gefällt, mit anderen Worten: Gerichte sind darauf bedacht, keine Urteile zu fällen, die das System gefährden würden. 104

Die Proliferation internationaler Gerichte steht außer Frage. ${ }^{105}$ Die Regel ist dabei die Staatenklage. Anders als außergerichtliche Verfahren sind die Entscheidungen der Gerichte verbindlich. Sie stellen einen Verstoß unwiderruflich fest und werden oftmals gleichermaßen in internationaler wie nationaler Öffentlichkeit wahrgenommen, ${ }^{106}$ was den Reputationsmechanismus stärkt. Andererseits allerdings wird der Reputationsmechanismus dadurch gedämpft, dass die Gerichtsbarkeit erst anerkannt werden muss. ${ }^{107}$ Nur einzelne Regime, wie die WTO oder auch der Menschenrechtsschutz in Europa, verlangen die obligatorische Gerichtsbarkeit, wenn ein Staat dem System beitritt. Zwar sind Staaten verpflichtet, einem Gerichtsurteil nachzukommen, aber auch hier können sich Durchsetzungsschwierigkeiten ergeben. Denn Gerichte allein effektuieren Völkerrecht nicht, vielmehr müssen die Urteile be-

103 C. Gray, Judicial Remedies in International Law, 1990; G. Guillaume, The Future of International Judicial Institutions, 44 International and Comparative Law Quarterly (1995), S. 848; K. Highet/ P.H.F. Bekker/R.P. Alford, International Courts and Tribunals, 31 International Lawyer (1997), S. 599; M.W. Janis, (Hrsg.), International Courts for the Twenty-First Century, 1992, S. 13; R. Jennings, The Judicial Enforcement of International Obligations, 47 ZaöRV 1987, S. 3; K.J. Alter, Do International Courts Enhance Compliance with International Law?, 25 Review of Asian and Pacific Studies 2003, S. 51.

104 A. Stone Sweet/T. Brunell, The European Court of Justice, State Noncompliance, and the Politics of Override, 106 American Political Science Review 2012, S. 204; C. Carrubba, Courts and Compliance in International Regulatory Regimes, 67 The Journal of Politics 2005, S. 669; C. Carrubba/M. Gabel/ C. Hankla, Understanding the Role of the European Court of Justice in European Integration, 106 American Political Science Review 2012, S. 214.

105 C.P.R. Romano, The Proliferation of International Judicial Bodies: The Pieces of the Puzzle, 31 New York University Journal of International Law and Politics 1998, S. 709.

106 Selbiges gilt damit auch für Gutachten internationaler Gerichte.

107 Wichtigstes Beispiel ist der IGH, dem aber die Staaten auch nur durch besonderen Einwilligungsakt unterworfen sind, vgl. Art. 36 (2) IGH-Statut. 
folgt werden, weshalb sich die Frage stellt, warum und unter welchen Umständen Staaten Urteile befolgen.

So ist die vor dem IGH obsiegende Partei bei Nichtbefolgung des Urteils durch die Gegenpartei zwar berechtigt, den Sicherheitsrat anzurufen, der Zwangsmaßnahmen verhängen kann. Dennoch aber kann zumindest jede Vetomacht durch die Zusammensetzung des Sicherheitsrats eine Durchsetzung gegen sich verhindern. Trotzdem werden die Urteile in aller Regel umgesetzt, ${ }^{108}$ was auf Reputationseffekte oder, wenn Gegenmaßnahmen ergriffen werden, auf Reziprozitätseffekte zurückzuführen ist.

Oftmals sind auch dann Staatenklagen vorgesehen, wenn Nutznießer der Norm eigentlich Individuen oder Unternehmen sind. Diese können informell auf eine Klage drängen. Ob daraufhin tatsächlich geklagt wird, hängt von diversen - auch diplomatischen - Erwägungen ab: Regierungen agieren als "political filter" ${ }^{109}$ bzw. "gatekeeper".

In Ausnahmefällen sehen Staaten aber auch interne Mechanismen vor, die es den Normbetroffenen ermöglichen, den Staat anzuhalten, eine Staatenklage anzustrengen. Damit wird das Völkerrecht bereits im Vorfeld innerstaatlich effektuiert; erstens, indem die Normbetroffenen den Staat über Völkerrechtsverstöße informieren und zweitens durch ein Verfahren, in welchem sich der Staat rechtfertigen muss, weshalb ein vermeintlicher Völkerrechtsverstoß nicht durch Klage geahndet wird. Solche Verfahren sehen die EU und die USA bei behaupteten Verstößen gegen das Welthandelsrecht vor. ${ }^{110}$ Je höher die Chance ist, dass ein Verfahren angestrengt wird, desto höher ist auch der Anreiz für die privaten Akteure, ihren Regierungen potentielle Verstöße anderer Staaten zu melden. Auf diese Weise werden sie als Informationssammler genutzt; eine Aufgabe, die staatliche Stellen nicht befriedigend bewältigen können.

108 C.-A. Fleischhauer, Der Internationale Gerichtshof und die Staatengemeinschaft am Ende des Jahrhunderts, 74 Die Friedens-Warte 1999, S. 119. Im Nicaragua Fall (Fn. 18), haben die Vereinigten Staaten die Durchsetzung allerdings durch ein Veto im Sicherheitsrat verhindert.

109 A.O.N. Sykes, Public vs. Private Enforcement of International Economic Law: Of Standing and Remedy, 34 Journal of Legal Studies 2005, S. 631 (S. 647 f.).

110 Siehe für die EU Verordnung (EG) Nr. 3286/94 des Rates vom 22. Dezember 1994 zur Festlegung der Verfahren der Gemeinschaft im Bereich der gemeinsamen Handelspolitik zur Ausübung der Rechte der Gemeinschaft nach internationalen Handelsregeln, insbesondere den im Rahmen der Welthandelsorganisation vereinbarten Regeln, Abl. Nr. L 349 vom 31/12/1994, S. 71. Für die USA siehe M. Bronckers, Private Appeals to WTO Law - An Update, in: C. Baudenbacher/E. Busek (Hrsg.), The Role of International Courts, Germany: German Law Publishers 2008, S. 117 und G.C. Shaffer, Defending Interests - Public-Private Partnerships in WTO Litigation, Washington D.C.: Brookings Institution Press 2003. Für China J. Song, A Comparative Study on the Trade Barriers Regulation and the Foreign Trade Barriers Investigation Rules, 41 Journal of World Trade 2007, S. 799. 


\section{c) Verfahren mit Individuen}

Die Mediatisierung des Individuums im Völkerrecht wird immer weiter aufgeweicht oder, umgekehrt ausgedrückt: die Stellung des Individuums wird aufgewertet auf zweierlei Weise. Zum einen kann sich ein Individuum wegen Völkerrechtsverbrechen vor einem internationalen Gericht zu verantworten haben, zum anderen kann ein in seinen Rechten verletztes Individuum sein Recht selbständig einklagen.

Die Errichtung des Internationalen Strafgerichtshof (ICC) durch das Rom-Statut war insoweit ein Meilenstein, als nunmehr über Völkerrechtsverbrechen auch regelmäßig und nicht nur ad hoc auf internationaler Ebene geurteilt werden kann. ${ }^{111}$ Dadurch wird eine neue Ebene des Strafrechts geschaffen und - trotz der Subsidiarität - den Staaten die Strafgewalt, eine klassische Domäne der Souveränität, teilweise entzogen. Dadurch können auch mögliche Defizite von Willen und Können der Durchsetzung auf nationaler Ebene gemildert werden. Das Völkerstrafrecht richtet sich zudem an die Rechtsunterworfenen, d.h. die potentiellen Straftäter, und will so auch Anreizwirkungen entfalten. Zudem arbeitet es über Transparenz und Reputationseffekte von Unrechtsurteilen. Es ist zudem paradigmatisch für die Auflösung der strengen konzeptionellen Grenzen im Völkerrecht: Effektuierung erfolgt nicht mehr nur zwischenstaatlich, sondern auch durch direkten Zugriff auf Individuen unter (teilweiser) Ausschaltung nationaler Gerichte. ${ }^{112}$

Kommen wir nun zu der Ermächtigung von Individuen, ihre Rechte vor internationalen Streitbeilegungsinstanzen selbst wahrzunehmen. Individualklagen im Völkerrecht sind immer noch selten. Sie haben den Vorteil, dass sie die jeweils Betroffenen dazu ermächtigen, das Recht in die eigenen Hände zu nehmen. Der Anreiz, das Recht in Anspruch zu nehmen, ist daher erheblich höher, als wenn Staaten das Recht eigener (oder fremder) Staatsbürger gerichtlich durchsetzen sollen. Denn aus einer Rational-Choice Perspektive besitzt ein Staat nur beschränkt Anreize, fremde Rechte durchzusetzen, selbst wenn es sich um die Rechte eigener Staatsbürger handelt. Individualklagen haben darüber hinaus den Vorteil, dass sie Völkerrechtsverletzungen ans Licht bringen und autoritativ eine Völkerrechtsverletzung feststellen. Damit wird dem “managerial approach" Rechnung getragen, der insbesondere auch die Unsicherheit über die Rechtslage thematisiert.

Die bekanntesten Individualklagemöglichkeiten finden sich im regionalen Menschenrechtsschutz. Zu nennen ist hier auch die Möglichkeit, vor den Komitees der globalen Menschenrechtsverträge Beschwerde zu erheben und dadurch deren nicht-

111 Umfassend dazu G. Werle, Völkerstrafrecht, Tübingen 2012 sowie von Arnauld, Völkerrecht (Fn. ), S. $504 \mathrm{ff}$.

112 So auch etwa die Terrorismusbekämpfung durch den Sicherheitsrat und sein Counter-Terrorism Committee (CTC), geschaffen durch Resolution 1373 (2001) vom 28. September 2001; vgl. ausführlich E. Rosand, Security Council Resolution 1373, the Counter-Terrorism Committee, and the Fight against Terrorism, 97American Journal of International Law 2003, S. 333. 
bindende Auffassungen zur Rechtslage zu erlangen. ${ }^{113}$ Alle Menschenrechtsverträge sehen auch Staatenklagen vor. Aus Rational-Choice Sicht ist es nicht verwunderlich, dass diese kaum genutzt werden: Staaten klagen nur dann, wenn die Rechte ihrer eigenen Minderheiten in anderen Staaten verletzt werden. ${ }^{114}$ Damit ist das Menschenrechtssystem zur gerichtlichen Durchsetzung primär auf die Initiative der Betroffenen angewiesen. Und diese müssen ihrerseits erhebliche Kosten auf sich nehmen, um ihr Recht durchzusetzen. Der Grund hierfür liegt zum einen in der nationalen Rechtswegerschöpfung, die materielle und immaterielle Kosten (in autoritären Staaten auch politische Repressalien gegen Kläger und Anwälte) mit sich bringt. Hinzu kommt, dass sich auch bei manchen Menschenrechten ein Kollektivhandelsproblem ergibt. Betrifft die Klage z.B. Gruppenrechte (etwa in Bezug auf sexuelle Orientierung, Minderheitenrechte oder Religionsfreiheit), dann klagt der Kläger faktisch nicht nur für sich selbst (auch wenn er alle Kosten trägt), sondern für die gesamte Gruppe, die von einem Urteil zu seinem Gunsten profitiert. ${ }^{115}$ Damit entsteht ein Trittbrettfahrerproblem. Abhilfe hierfür, wie auch für das Kostenproblem schaffen einerseits Prozesskostenhilfen oder die Zulassung von altruistischen NGO-Klagen andererseits. Manche der regionalen Menschenrechtsregime lassen genau dies zu.

Sehr viel effektiver sind dagegen Klagen im internationalen Investitionsschutzrecht; ${ }^{116}$ dem zweiten Bereich, in welchem Individualklagen zugelassen werden. In aller Regel wird hier auf die nationale Rechtswegerschöpfung verzichtet; der Kläger kann seine Rechte direkt vor einem internationalen Schiedsgericht geltend machen. Da zudem die Kläger oft große Unternehmen sind, spielt die Kostenfrage keine so große Rolle. Wird zugunsten des Klägers auf Schadensersatz erkannt, ist das Schiedsurteil gemäß Art. 54 der ICSID Konvention in allen Vertragsstaaten wie ein letztinstanzliches Urteil ohne Überprüfung vollstreckbar. Für die Durchsetzung kann dann auf nationale Durchsetzungsinstanzen zurückgegriffen werden, was das Investitionsschutzrecht sehr effektiv macht, denn die klassische Staat-zu-Staat Durchsetzung wird umgangen: Urteile können direkt mittels nationaler Zwangsvollstreckungs-

113 Bereits die Ratifikation des Vertrages und die getrennte Zustimmung zur individuellen Beschwerdemöglichkeit ist zahlenmäßig sehr unterschiedlich. Während 167 Staaten den IPBürg ratifiziert haben, lassen nur 114 Staaten Individualbeschwerden nach dem 1. Zusatzprotokoll zu. Ein ähnliches Bild ergibt sich bei den anderen globalen Verträgen auf den Websites der Verträge.

114 Umfassend A. van Aaken, Making International Human Rights Protection More Effective: A Rational-Choice Approach to the Effectiveness of Ius Standi Provisions, in: S. Voigt/M. Albert/D. Schmidtchen (Hrsg.), International Conflict Resolution, Conferences on New Political Economy 23, Tübingen 2006, S. 29.

115 Früh dazu R. v. Jhering, Der Kampf um's Recht, Wien: Manz 1880, S. 49.

116 Übersichtshalber R. Dolzer/C. Schreuer, Principles of International Investment Law, Oxford: Oxford University Press 2008. 
maßnahmen gegen die Staaten vollstreckt werden. ${ }^{117}$ Hinzu kommt die Veröffentlichung der Verfahren bei ICSID, durch welche sich der (negative) Reputationseffekt für die verklagten Staaten erhöht. Dies kann zur Abnahme ausländischer Direktinvestitionen führen, da Investoren die Reputation hoher politischer Risiken mit weniger Investitionen "strafen”. Der Kapitalmarkt effektuiert auf diese Weise das internationale Investitionsschutzrecht. ${ }^{118}$ Es sei angemerkt, dass der Reputationsfaktor sich hier auf die Reaktionen privater Akteure auswirkt, nicht auf Staaten.

\section{Monitoring und Reporting}

Um den Reputationsmechanismus im Völkerrecht zu effektuieren, sind Monitoring und Reporting eine notwendige Bedingung. ${ }^{119}$ Auch solche Verträge, die einen gerichtlichen Streitbeilegungsmechanismus nicht vorsehen, haben in aller Regel solche Mechanismen, sozusagen faute de mieux. Nur wenn Informationen zu Verstößen vorliegen, können die Effektuierungsmechanismen spielen; unabhängige Expertengremien machen die Information gewichtiger und klarer. Gerade in Verträgen, die öffentliche Güter betreffen (wie im Umwelt- und Menschenrechtsschutz), ${ }^{120}$ werden regelmäßig Staatenberichte verlangt. ${ }^{121}$ Häufig allerdings leiden diese Berichte unter verzögerter Einreichung und Unvollständigkeit. Dem wurde Abhilfe geschaffen, indem nun auch Berichte von NGOs akzeptiert werden. ${ }^{122}$ Damit wird ein erheblicher Druck auf Staaten ausgeübt, ihren Verpflichtungen nachzukommen: die Rolle der NGOs im Monitoring von Völkerrechtsverstößen ist gut analysiert. ${ }^{123}$ NGOs doku-

117 G. Born, A New Generation of International Adjudication, 61 Duke Law Journal 2012, S. 775. Voraussetzung ist allerdings, dass Vermögen in anderen Staaten nicht der Immunität unterliegt. Siehe dazu A. van Aaken, Blurring Boundaries between Sovereign Acts and Commercial Activities. A Functional View on Regulatory Immunity and Immunity from Execution, in: A. Peters/E. Lagrange/ S. Oeter (Hrsg.), Immunities in the Age of Global Constitutionalism, Leiden: Brill 2013, erscheint demnächst.

118 T. Allee/C. Peinhardt, Contingent Credibility: The Impact of Investment Treaty Violations on Foreign Direct Investment, 65 International Organization 2011, S. 401.

119 T. König/L. Mäder, The Strategic Nature of Compliance: An Empirical Evaluation of Law Implementation in the Central Monitoring System of the European Union, American Journal of Political Science 2013, online Version unter: http://onlinelibrary.wiley.com/doi/10.1111/ajps.12038/pdf (zuletzt besucht am 25. August 2013).

120 E. Klein (Hrsg.), The Monitoring System of Human Rights Treaty Obligations, Berlin: Berlin-Verl. Spitz 1998 sowie T. Treves, Introduction to Non-compliance Procedures and Mechanisms and the Effectiveness of International Environmental Agreements, in: T. Treves/A. Tanzi/L. Pineschi (Hrsg.), Non-compliance Procedures and Mechanisms and the Effectiveness of International Environmental Agreements, Cambridge: Cambridge University Press 2009, S. 1 ff. sowie A. Tanzi/C. Pitea, Lessons Learned and the Way Forward, in: T. Treves/A. Tanzi/L. Pineschi (Hrsg.), Non-compliance Procedures and Mechanisms and the Effectiveness of International Environmental Agreements, Cambridge: Cambridge University Press 2009, S. 569 ff. (573-74, 578).

121 B. Simma, The Examination of State Reports, in: E. Klein (Hrsg.), Monitoring (Fn. 120), S. 31.

122 Vgl. nur für die Anti-Folter-Konvention: http://www2.ohchr.org/english/bodies/cat/follow up ngo.htm (zuletzt besucht am 15. Mai 2013).

123 ILA, Non State Actors in International Law. Second Report of the Committee 2012, erhältlich unter http://www.ila-hq.org/en/committees/index.cfm/cid/1023 (zuletzt besucht am 15. Mai 2013). 
mentieren und veröffentlichen Verletzungen des Völkerrechts und verleihen so dem Reputationsmechanismus ein erhebliches Gewicht.

Auch „peer-monitoring“ effektuiert die Information; die Feststellung von Verstößen besitzt hier erhöhte Glaubwürdigkeit. So verwenden etwa der Europarat und die OECD im Bereich der Korruptionsbekämpfung (bei welcher erhebliche Anreize für Staaten bestehen, die internationalen Konventionen nicht pflichtgemäß umzusetzen) neben den Staatenberichten „peer-monitoring “ Verfahren, während andere AntiKorruptionskonventionen dies nicht vorsehen. ${ }^{124}$ Ebenso spielen IGOs durch die Bereitstellung einer Plattform für intergouvernementalen Austausch, durch „peerpressure“, Monitoring und technische Hilfe eine erhebliche Rolle. Besonders für das Umweltrecht ist dies von großer Bedeutung; der „managerial approach“ kommt hier zum Tragen. ${ }^{125}$

\section{Staatenverantwortlichkeit}

Staatenverantwortlichkeit, so wie sie in den ILC Articles wiedergegeben ist, ${ }^{126} \mathrm{knüpft}$ an staatliche Völkerrechtsverletzungen diverse Rechtsfolgen wie Wiedergutmachung und Schadensersatzzahlungen. Aber auch hier stellt sich wieder die Frage, wie solche Forderungen durchgesetzt werden können. Die ILC Articles setzen - durch die erlaubten Gegenmaßnahmen ${ }^{127}$ - auf dezentrale Durchsetzung. Normen werden damit weitgehend als selbstdurchsetzend gedacht: Die verletzenden Staaten werden durch Zufügung von Rechtsnachteilen zur Befolgung angehalten. Der verletzte Staat beantwortet die Völkerrechtsverletzung, indem er die eigenen Verpflichtungen suspendiert, diplomatische Beziehungen beendigt, ökonomische Sanktionen verhängt oder indem er dem verletzenden Staat über Druck und Beschämung soziale Kosten auferlegt. ${ }^{128}$ Gegenmaßnahmen sind zum einen Retorsionen, d.h. Reaktionen mit völkerrechtlich erlaubten Mitteln. Darunter fallen z.B. die Einstellung von Entwicklungshilfe oder der Nichtabschluss von Verträgen. ${ }^{129}$ Zweitens erlauben die ILC Articles Repressalien. Hier ergreift ein Staat gegen den verletzenden Staat an sich völkerrechtswidrige Maßnahmen, indem er etwa die Erfüllung von Verträgen verweigert. Grenzen ergeben sich aus Repressalienverboten, also zwingenden Normen des Völkerrechts sowie Menschenrechten und sog. self-contained regimes. Während ers-

124 Vgl. A. van Aaken, Die UN-Konvention gegen Korruption: Alter Wein in neuen Schläuchen?, in: R. Hofman/C. Pfaff (Hrsg.), Die Konvention der Vereinten Nationen zur Bekämpfung der Korruption, Baden-Baden 2006, S. 9.

125 Vgl. Boyle, Saving (Fn. 100).

126 Vgl. GA Res. 56/83, 28. Januar 2002, Annex Responsibility of States for Internationally Wrongful Acts (ILC Articles).

127 Siehe zu Retorsion und Repressalie C. Tomuschat: Repressalie und Retorsion, zu einigen Aspekten ihrer innerstaatlichen Durchführung, 33 ZaöRV 1973, S. 179.

128 Schachter, Practice (Fn. 77), S. 185; N. White/A. Abass, Countermeasures and Sanctions, in: M.D. Evans (Hrsg.), International Law, Oxford: Oxford University Press 2006, S. 509.

129 Vgl. Vöneky, Durchsetzung (Fn. 3), S. 490. 
tere beiden als normative öffentliche Güter bezeichnet werden können, die nicht durch Selbsthilfe geschädigt werden sollen, nimmt letzteres Verbot solche Regime aus, die von einer speziellen Reziprozität profitieren, indem sie ihre eigenen Durchsetzungsmechanismen haben (WTO). In allen übrigen Gebieten stärkt so das Völkerecht den Reziprozitätsmechanismus.

Probleme ergeben sich bei der Verletzung sog. erga omnes Verpflichtungen, also Pflichten "of a state towards the international community as a whole". ${ }^{130}$ Bei erga omnes-Pflichten wird angenommen, dass sie im Interesse der Völkerrechtsgemeinschaft bestehen und alle Staaten insofern ein rechtliches Interesse an ihrer Einhaltung haben. Beispiele sind das Aggressions- und Genozidverbot, wie auch grundlegende Menschenrechte. Strukturell liegt hier ein rechtlich gekorenes öffentliches Gut vor. Damit stellt sich auch die Frage, wer ein Interesse an der Durchsetzung hat. Idealerweise sollten kollektive Durchsetzungsmechanismen zum Tragen kommen. Allerdings stellt sich in diesem Zusammenhang abermals die o.g. Trittbrettfahrerproblematik. Mithin ist eher eine zu geringe Durchsetzung zu erwarten, was dafür spricht, allen Staaten bei Verstößen gegen inter omnes Verpflichtungen Repressalien zu erlauben. ${ }^{131}$ Dagegen spricht andererseits die Möglichkeit eines Missbrauchs durch starke Staaten. Die ILC Articles differenzieren daher die Gegenmaßnahmen je nach Betroffenheit. ${ }^{132}$

\section{Verlinkung von Verträgen}

Befolgung des Völkerrechts kann auch dadurch induziert werden, dass manche Staaten dafür „zahlen“, dass andere Staaten Völkerrecht einhalten. Dies erfolgt oftmals durch die Verlinkung von Verträgen, die auf den ersten Blick sachlich voneinander unabhängig sind, ${ }^{133}$ also z.B. von Wirtschaftsverträgen mit Verträgen über Umweltbzw. Menschenrechtsfragen. Ebenso enthalten asymmetrische Sicherheitsabkommen teilweise Handelsnormen. ${ }^{134}$ Auch wird die Einhaltung von Menschenrechtsverträgen in ökonomischen Abkommen (insbesondere in Freihandelsabkommen) teilweise als wesentliche Vertragsverpflichtung aufgenommen, um so im Verletzungsfalle Reaktionsmöglichkeiten im Hinblick auf den Wirtschaftsvertrag zu eröffnen. ${ }^{135}$ In gleichem Sinne verlangen einseitig gewährte Zollpräferenzen für Entwicklungsländer als

130 IGH, Barcelona Traction, Light and Power Company Limited, ICJ Reports 1970, S. 2 ff.

131 So auch die überwiegende Auffassung, vgl. A. Verdross/B. Simma, Universelles Völkerrecht, Berlin 1984, S. 909.

132 Art. $42 \mathrm{~b}$ und 48 ILC Articles.

133 Auf den ersten Blick, da auf den zweiten Blick Umweltstandards und Arbeitsrechte (als eine Art der Menschenrechte) zu den Wettbewerbsbedingungen gehören, unter denen Staaten produzieren.

134 P. Poast, Can Issue Linkage Improve Treaty Credibility? Buffer State Allliances as a 'Hard Case', Journal of Conflict Resolution, online first version: DOI: 10.1177/0022002712449323 2012, findet dadurch höhere Befolgungsraten.

135 Vgl. etwa das Cotonou-Abkommen zwischen den AKP-Staaten und der EG (Abkommen von Cotonou, ABl. 2000 Nr. L 317, S. 3), Art. 9 Abs. 2 UAbs. 4. Die USA sehen ähnliche Bedingungen vor in den lateinamerikanischen Abkommen. 
Bedingung die Ratifikation von Menschenrechts-, Umweltschutz- oder anderen Verträgen, wie etwa die UN Konvention gegen Korruption. ${ }^{136}$ Während Handelsverträge durch das Reziprozitätsprinzip selbstdurchsetzend sind, sind es Umweltverträge und Menschenrechtsverträge aufgrund ihres Charakters als öffentliches Gut nicht. Durch die Verlinkung wird das Reziprozitätsprinzip als Durchsetzungsmechanismus ausgeweitet. Die Kosten der Nichtbefolgung eines Vertrages werden erhöht, falls eine Verlinkung zu einem anderen, wichtigen Sachgebiet besteht. Solche Verlinkungen können auch innerhalb von “Großverträgen” (etwa der WTO) bestehen, die „crossretaliation“ zwischen einzelnen Abkommen vorsehen. ${ }^{137}$

\section{Dezentrale Durchsetzung auf nationaler Ebene}

Staaten bzw. Regierungen spielen immer auf zwei Ebenen: zwischenstaatlich und innerstaatlich. Viele der völkerrechtlichen Verpflichtungen müssen innerstaatlich umgesetzt werden; letztendliche Normadressaten sind oftmals Private, etwa im Umweltschutzbereich, bei der Geldwäsche oder im Korruptionsbereich. Effektuiert wird das Völkerrecht hier auf zweierlei Art: zum einen über nationale Gerichte (1.), zum anderen über interne politische Prozesse (2.).

\section{Nationale Gerichte}

In den Rechtsgebieten, in denen zwischenstaatliche Reziprozität nicht spielt, sind die nationalen Gerichte zur Durchsetzung des Völkerrechts umso wichtiger. ${ }^{138}$ Paradebeispiel sind die Menschenrechte. ${ }^{139}$ Staaten, in denen Völkerrecht direkt anwendbar ist und unmittelbare Wirkung hat, verwenden ihre nationalen Gerichte zur Durchsetzung - und damit auch die ganze Kraft des nationalen Gewaltmonopols. Diese

136 Vgl. etwa die Zollpräferenzen (generalized system of preferences) der EU und der USA, welche die Einhaltung von Menschenrechtsstandards und Umweltstandards vorsehen. Vgl. M. Snyder, GSP and Development. Increasing the Effectiveness of Nonreciprocal Preferences, 330 Michigan Journal of International Law 2011/12, S. 821; UNCTAD, Generalized System of Preferences, Handbook on the Scheme of the European Community; U.N. Doc. UNCTAD/ITCD/TSB/Misc.25/Rev. 3, 2008; E.M. Hafner-Burton, Trading Human Rights: How Preferential Trading Agreements Influence Government Repression, 59 International Organization 2005, S. 593.

137 Art. 22 (3) DSU.

138 R. Falk, The Role of Domestic Courts in the International Legal Order, New York: Syracuse University Press 1964; H. Schermers, The Role of Domestic Courts in Effectuating International Law, 3 Leiden Journal of International Law 1990, S. 77; T. Franck/G. Fox (Hrsg.), International Law Decisions in National Courts, New York: Transnational Publishers, 1996; B. Conforti, International Law and the Role of Domestic Legal Systems, London: Martinus Nijhoff Publishers, 1993; H. Koh, The 1998 Frankel Lecture: Bringing International Law Home, 35 Houston Law Review 1998-1999, S. 623.

139 B. Conforti/F. Francioni (Hrsg.), Enforcing International Human Rights in Domestic Courts, Boston: Martinus Nijhoff Publishers 1997; H. Koh, How is International Human Rights Law Enforced?, 74 Indiana Law Journal 1998-1999, S. 1397. 
Art der Durchsetzung wird in der Rechtswissenschaft weithin diskutiert, ${ }^{140}$ denn dank des Zugangs zu Gericht und nationaler Durchsetzung wird das Völkerrecht unmittelbar effektuiert. Allerdings sind auch hier einige Probleme zu beachten. Zum einen ist der Stellenwert des Völkerrechts in der nationalen Rechtsordnung relevant. In dualistischen Systemen, wo es der Umsetzung von völkerrechtlichen Normen in nationales Recht bedarf, besitzen die Staaten ein gewisses Ermessen, während in monistischen Staaten Völkerrecht grundsätzlich ohne Umsetzungsakt als nationales Recht gilt und internationale Menschenrechte teilweise gar Verfassungsrang innehaben (dies ist idealtypisch formuliert; es besteht Einigkeit darüber, dass der Monismus in seiner Reinform von Staaten nicht praktiziert wird). ${ }^{141}$

Aber nicht in allen Staaten werden Gerichtsurteile umgesetzt und nicht in allen Rechtsordnungen sind Gerichte unabhängig. Dies kann dazu führen, dass die Durchsetzung leidet, selbst wenn Völkerrechtsnormen in die nationale Rechtsordnung inkorporiert sind. Ein weiteres Problem ergibt sich, wenn es sich nicht um Individualrechte handelt, sondern um den Schutz öffentlicher Güter, weil dann oft keine Klagebefugnis besteht und damit kein Zugang zu innerstaatlichen Gerichten eröffnet wird. Dies betrifft etwa das Umweltrecht. Erkannt wurde die Problematik in der Aarhus-Konvention, ${ }^{142}$ welche zur Durchsetzung gerade den Zugang zu Gericht auch für Verbände verlangt und sich damit des innerstaatlichen Effektuierungsmechanismus bedient. Eine generelle Ausweitung der Klagebefugnis würde auch anderen Völkerrechtsgebieten zu größerer Effektuierung verhelfen.

\section{Nationale politische Prozesse}

Gerade im Menschenrechtsbereich wurden die nationalen politischen Effektuierungsprozesse bereits gut untersucht. ${ }^{143}$ Diskutiert wird hier insbesondere die sog.

140 Vgl. Lord Bingham, Foreword, in: S. Fatima (Hrsg.), Using International Law in Domestic Courts, Oxford: Hart Publishing 2005. "To an extent almost unimaginable even thirty years ago, national courts in this and other countries are called upon to consider and resolve issues turning on the correct understanding and application of international law, not on an occasional basis, now and then, but routinely, and often in cases of great importance." Siehe auch A.E. Roberts, Comparative International Law? The Role of National Courts in International Law, 60 International \& Comparative Law Quarterly 2011, S. 57 m.w.N.

141 Zum Diskussionsstand in Bezug auf nationale Gerichte siehe F. Francioni, Judicial Models of International Law Enforcement, in: F. Cafaggi (Hrsg.), Enforcement (Fn. 2), S. 167.

142 Art. 9 Übereinkommen der Wirtschaftskommission für Europa (UNECE) über den Zugang zu Informationen, die Öffentlichkeitsbeteiligung an Entscheidungsverfahren und den Zugang zu Gerichten in Umweltangelegenheiten. Vgl. auch C. Walter, Internationalisierung des deutschen und europäischen Verwaltungsverfahrens- und Verwaltungsprozeßrechts - am Beispiel der Aarhus-Konvention, 40 EuR 2005, S. 302.

143 Vgl. für die internationale Ebene Finnemore/Sikkink, Dynamics (Fn. 61); Simmons, Mobilizing (Fn. 8); T. Risse, International Norms and Domestic Change: Arguing and Communicative Behavior in the Human Rights Area, 27 Politics and Society 1999, S. 529; Risse/Sikkink, The Socialization of International Human Rights Norms into Domestic Practices: Introduction, in: T. Risse/S.C. Ropp/K. Sikkink (Hrsg.), Norms (Fn. 61). 
Normspirale. ${ }^{144}$ Ausgangspunkt waren konstruktivistische Ansätze, welche die Beziehungen zwischen Ideen und sozialen Prozessen beobachten. ${ }^{145}$ Betrachtet werden Normendynamiken, um zu beantworten, wie normativer Wandel beeinflusst werden kann und welche Strategien (blaming, shaming, framing, norm advocacy) zum Erfolg führen. Drei verschiedene Sozialisierungsprozesse bewirken die Internalisierung von Normen (womit Normen zum Handlungsgrund werden; internalisierte Regelbefolgung): instrumentelle Adaptation, Argumentation und Habitualisierung. Das Modell beinhaltet fünf Phasen: Erstens, die Repressionsphase, in der in einem Land Menschenrechte missachtet werden. Zweitens, die Negierung der Menschenrechtsverletzungen (denial) durch die Regierung angesichts internationaler Proteste und die Weigerung, sich internationaler Gerichtsbarkeit zu unterwerfen. Drittens, „low cost“ taktische Konzessionen, mit denen versucht wird, die Menschenrechtsbewegung zu beruhigen sowie ggf. die Ratifikation von Verträgen. In dieser Phase kann die Regierung die Bewegung entweder unterdrücken oder ihr weitere Konzessionen machen (instrumentelle Phase). Viertens, die präskriptive Phase, in der die Ratifizierung der Verträge und die Umsetzung in das nationale Recht erfolgt. Fünftens, normkonsistentes Verhalten; die Vertragsbindung stärkt Menschenrechtsbefürworter gegenüber den Menschenrechtsgegnern, und es kommt zur normgeleiteten "logic of appropriateness".

Soweit die Theorie. Frühe empirische Forschung zu Menschenrechtsverträgen fand keinen statistisch signifikanten Kausalzusammenhang zwischen Vertragsratifikation und Menschenrechtslage. ${ }^{146}$ Die neuere Forschung konzentriert sich auf die zuvor vernachlässigten Kausalmechanismen, die von "commitment" $\mathrm{zu}$ "compliance" führen: u.a. Diskurse, die durch Eliten initiiert werden, rechtliche Verfahren (die Gerichte, s.o.) sowie politische Mobilisation. ${ }^{147}$ Dies gilt allerdings nur bedingt: weder bei stabilen Demokratien, noch bei stabilen Autokratien kann ein signifikanter Kausaleffekt zwischen Ratifikation (commitment) und Menschenrechtslage festgestellt werden. Die größten Effekte sind in Transformationsstaaten zu finden. ${ }^{148}$

144 Zuerst M.E. Keck/K. Sikkink, Activist Beyond Borders. Advocacy Networks in International Politics, Ithaca, NY: Cornell University Press 1998, sodann Risse/Ropp/Sikkink (Hrsg.), Norms (Fn. 61); nun T. Risse/S.C. Ropp/K. Sikkink (Hrsg.), The Persistent Power of Human Rights. From Commitment to Compliance, Cambridge: Cambridge University Press 2013.

145 Vgl. etwa A.P. Cortell/J. Davis, How Do International Institutions Matter? The Domestic Impact of International Rules and Norms, 40 International Studies Quarterly 1996, S. 451.

146 O.A. Hathaway, Do Human Rights Treaties Make a Difference?, 111 Yale Law Journal 2002, S. 1935; E. Neumayer, Do International Human Rights Treaties Improve Respect for Human Rights?, 49 Journal of Conflict Resolution 2005, S. 925.

147 Risse/Ropp/Sikkink (Hrsg.), Power (Fn. 144), S. 11; Simmons, Mobilizing (Fn. 8).

148 B. Simmons, From Ratification to Compliance: Quantiative Evidence on the Spiral Model, in: T. Risse/S.C. Ropp/K. Sikkink (Hrsg.), Power (Fn. 144), S. 43. 


\section{Transnationale Mechanismen zur Effektuierung des Völkerrechts}

Neben den traditionell diskutierten Mechanismen zeichnen sich neuere Entwicklungen ab, die erstens vermehrt nichtstaatliche Akteure in die Effektuierung des Völkerrechts einbeziehen und sich zweitens vermehrt soft law Instrumente zu Nutze machen. Die Flut von soft law-Normen im internationalen Bereich, seien sie staatlich, nichtstaatlich oder in Kooperation entstanden, ist empirisch dokumentiert: der Abschluss völkerrechtlicher Verträge nimmt in der Tendenz ab, soft law nimmt zu. ${ }^{149}$ Das "public ordering" wird durch "private ordering" bzw. "hybrid ordering" ergänzt. ${ }^{150}$ Gut dokumentiert ist die Rolle der nichtstaatlichen Akteure im Bereich der Normsetzung. ${ }^{151}$ Hier kreieren IGOs, Unternehmen (oder ihre Verbände) und NGOs Normen, oftmals auch in Kooperation, die dann globale Geltung erlangen: "we observe complex blends of private and public governance". ${ }^{152}$ Die Untersuchung der Durchsetzung der TPR ${ }^{153}$ ist bei weitem noch nicht so fortgeschritten wie im Völkerrecht. ${ }^{154}$ Bislang aber kann festgestellt werden, dass auch hier die (schieds-)gerichtliche Durchsetzung primär auf nationalstaatlicher Ebene eine große Rolle spielt, etwa bei der Durchsetzung privatrechtlicher Verträge, die TPR beinhalten und regulatorische Elemente aufweisen (etwa in Handelsketten). TPR stellt häufig soft law dar, kann aber dadurch in "hard law" transformiert werden. Diese Möglichkeit indes soll an dieser Stelle nicht weiterverfolgt werden. ${ }^{155}$ Vielmehr liegt der Schwerpunkt an dieser Stelle auf den nicht-juristischen, bisher vernachlässigten Durchsetzungsmechanismen. Hier sollen zwei herausgegriffen werden: zum einen

149 S. Voigt, The Economics of Informal International Law - An Empirical Assessment, in: J. Pauwelyn / R. Wessel/J. Wouters (Hrsg.), Informal International Lawmaking, Oxford: Oxford University Press 2012, S. 81.

150 Siehe umfassend $O E C D$, International Regulatory Co-operation: Addressing Global Challenges, Paris: OECD Publishing 2013. TPR "responds to the difficulties in co-ordinating the inconsistencies between standard setting and enforcement, the divergences between administrative and judicial enforcement, and asymmetries of information, which emerge at the transnational level and make purely public regulatory co-operation an insufficient response." (64f.).

151 A. Peters/L. Koechlin/T. Förster/G. Fenner Zinkernagel (Hrsg.), Non-State Actors as Standard Setters, Cambdrige: Cambridge University Press 2009.

152 K.W. Abbott/D. Snidal, International 'Standards' and International Governance, 8 Journal of European Public Policy 2001, S. 345 (346) sowie dies., International Regulation without International Government: Improving IO Performance through Orchestration, 5 Review of International Organizations Law Review 2010, S. 315.

153 OECD, International Regulatory Co-operation: Addressing Global Challenges, Paris: OECD Publishing 2013, S. 65: TPR "schemes vary enormously in terms of composition, mission, geographic reach, governance structures, and regulatory functions. The most common forms of regulatory output produced by TPR schemes are codes of conduct, guidelines, commercial contract schemes, industry standards and social or environmental standards".

154 Vgl. F. Cafaggi, Introduction, in: F. Cafaggi (Hrsg.), Enforcement (Fn. 2), S. 1 (4).

155 Vgl. aber ausführlich dazu OECD, International Regulatory Co-Operation: Rules for a Global World. GOV/RPC(2012)8/REV1 2013 erhältlich unter: <http://search.oecd.org/officialdocuments/ publicdisplaydocumentpdf/?cote=GOV/RPC(2012)8/REV1\&docLanguage=En $>$ (zuletzt besucht am 15. Mai 2013), sowie Cafaggi (Hrsg.), Enforcement (Fn. 2). 
Marktmechanismen (IV.), zum anderen die Netzwerkbildung (V.), bei welcher Normen über die Schaffung von Clubgütern effektuiert werden. ${ }^{156}$

\section{Nutzung des Marktmechanismus: Konsumentenpräferenzen}

Der Marktmechanismus ist als Effektuierungsinstrument des Völkerrechts weithin unbeachtet geblieben. Dennoch kann er sowohl für soft law als auch für klassisches Völkerrecht sehr effektiv sein. Insbesondere im Bereich der globalen öffentlichen Güter (Umwelt- und Menschenrechte) verspricht dieser Ansatz Komplementarität zu den klassischen Mechanismen. Letztere leiden aufgrund der oben dargestellten Anreizsituation oftmals an mangelnder zwischenstaatlicher Durchsetzung. Zudem gibt es zumindest im Umweltschutzbereich auf internationaler Ebene keine Möglichkeit zur Individualklage, ${ }^{157}$ da es an der notwendigen individuellen Betroffenheit fehlt. Im menschenrechtlichen Bereich werden die Rechte in "weak governance zones” nur unzureichend effektuiert; die Durchsetzung durch die betroffenen Akteure genügt nicht. ${ }^{158}$ Vermehrt werden aber Konsumentenpräferenzen als Effektuierungsmechanismus genutzt. ${ }^{159}$ Der Kimberley-Prozess, ${ }^{160}$ der den Handel mit Konfliktdiamanten erheblich besser einschränkte als die diversen Sanktionen des UN Sicherheitsrates, konnte nur effektiv sein, weil und solange die Konsumenten der wertvollen Schmuckdiamanten die Konfliktdiamanten boykottierten. Die Proliferation von Labelling Mechanismen und Zertifizierungsprozessen dient als Informationsgrundlage für Konsumentenkaufentscheidungen im menschenrechtlichen Bereich (etwa Kinderarbeit) ebenso wie im Umweltschutzbereich (etwa in der nachhaltigen Waldbewirtschaftung, Fischerei). Nur falls 1) die Konsumentenpräferenzen vorhanden sind (hier sei auf die o.g. Normenspiralen verwiesen), 2) diese marktmächtig genug sind, um bei Verstößen reale Umsatzeinbußen zu generieren und 3) die Labels glaubwürdig sind, kann davon ausgegangen werden, dass durch solche Mechanismen Völkerrecht effektuiert wird. Dies ist insbesondere in jenen Konstellationen wichtig, in denen

156 Umfassend dazu siehe A. van Aaken, Trust, Verify or Incentivize? Effectuating Public International Law Regulating Public Goods Through Market Mechanisms, 104th Proceedings of the American Society of International Law 2011, S. 153; A. van Aaken, Effectuating Public International Law through Market Mechanisms, 165 Journal of Institutional and Theoretical Economics 2009, S. 33.

157 Vgl. aber das World Bank Inspection Panel, vgl. www.worldbank.org/inspectionpanel (zuletzt besucht am 15. Mai 2013).

158 Vgl. nur UN High Commissioner of Human Rights, Guiding Principles on Business and Human Rights, 2011 sowie J. Ruggie, Report of the Special Representative of the Secretary-General on the Issue of Human Rights and Transnational Corporations and other Business Enterprises, UN Doc. A/ $\mathrm{HRC/8/5,} 7$ April 2008.

159 Daneben gelten natürlich die Corporate Social Responsibility Normen, die organisationsintern relevant sind, sowie mittlerweile auch die Kotierungsvorschriften für Publikumsgesellschaften im Bereich Konfliktrohstoffe, vgl. Section 1502 des Dodd-Frank Wall Street Reform and Consumer Protection Act (erhältlich unter: http://www.sec.gov/about/laws/wallstreetreform-cpa.pdf).

160 Kimberley Process Certification Scheme (KPCS). Zum Prozess siehe I.J. Tamm, Dangerous Appetites: Human Rights Activism and Conflict Commodities, 26 Human Rights Quarterly 2004, S. 687 sowie die Aufsätze von van Aaken (Fn. 156). 
Regierungen Völkerrecht innerstaatlich nicht umsetzen wollen oder können, wie es etwa im Fischereibereich der Fall ist. ${ }^{161}$ Angesetzt wird dann unmittelbar bei den Produzenten, die Anreize haben, ihre Produkte weiterhin zu verkaufen. Bekannt ist in diesem Zusammenhang etwa die Ineffektivität des Übereinkommens über den internationalen Handel mit gefährdeten Arten freilebender Tiere und Pflanzen (CITES). ${ }^{162}$ Grund für das Versagen des Abkommens sind u.a. Konsumentenpräferenzen für Produkte bedrohter Tierarten, wie etwa für Haifische, Rhinozerosse oder Tiger. ${ }^{163}$ Sind die interessierten Konsumenten kaufkräftig genug, bleiben die Gewinnspannen hoch - und der illegale Handel geht weiter. Können diese Präferenzen etwa durch Aufklärung oder „shaming“ beeinflusst werden, so würde der Markt und damit der Anreiz für die Ausrottung der Tiere durch Wilderer schwinden. Dieser Ansatz ist nur vor dem konstruktivistischen Hintergrund zu verstehen, bedarf aber gleichzeitig einer Rational-Choice Analyse zur Erklärung des Verhaltens der anderen beteiligten Akteure. Nur in Kombination von Interessen und Werten kann die Effektuierung der Normen erfolgen. ${ }^{164}$

Eine weitere Effektuierung, deren Hintergrund ebenfalls in den Konsumentenpräferenzen zu verorten ist, bedient sich privatrechtlicher Verträge, insbesondere Zuliefererketten: ${ }^{165}$ Zuliefererverträge werden verwendet, um die Einhaltung von Menschenrechtsstandards sicherzustellen. ${ }^{166}$ Selbiger Mechanismus kann auch für andere Normen, etwa für Umweltstandards, verwendet werden. ${ }^{167}$ Ein Verstoß in der Kette kann über die Reputation von Unternehmen dazu führen, dass diese empfindliche Umsatzeinbußen erleiden; die Effektuierung von Völkerrecht lässt sich insofern an Private auslagern.

161 So setzt etwa die International Commission for the Conservation of Atlantic Tunes regelmäßig die Fangquote oberhalb der von Experten empfohlenen Quote fest. NGOs wie der World Wildlife Fund dagegen verteilen Listen, auf denen gefährdete Fische aufgelistet sind, die dann dem Konsumenten ermöglichen, den Konsum solcher Fische selbst zu meiden.

162 IUCN, A Report by IUCN - The World Conservation Union on the Effectiveness of Trade Measures Contained in The Convention on International Trade in Endangered Species of Wild Fauna and Flora (CITES), 2000, http://www.cites.org/common/prog/economics/iucn-trademeasuresinCITES.pdf (zuletzt besucht am 15. Mai 2013).

163 Vgl. den UNEP Bericht für die 16. Vertragsstaatenkonferenz von CITES in Bangkok, März 2013: http://www.cites.org/eng/cop/16/doc/E-CoP16-07.pdf (zuletzt besucht am 15. Mai 2013).

164 In Bezug auf K.W. Abbott/D. Snidal, Values and Interests: International Legalization in the Fight Against Corruption, 31 Journal of Legal Studies 2002, S. 141.

165 F. Cafaggi, The Regulatory Functions of Transnational Commercial Contracts: New Architectures (August 26, 2012). Erhältlich bei SSRN: http://ssrn.com/abstract=2136632 (zuletzt besucht am 15. Mai 2013).

166 F. Cafaggi/A. Renda/R. Schmidt, Transnational Private Regulation, in: OECD (Hrsg.), International Regulatory Co-Operation: Rules for a Global World Vol. 1, 2003, erhältlich unter: <http://search.oecd.org/officialdocuments/publicdisplaydocumentpdf/?cote=GOV/RPC(2012)8/ANN6\&docLanguage=En $>$ (zuletzt besucht am 15. Mai 2013).

167 M.P. Vandenbergh, The New Wal-Mart Effect: The Role of Private Contracting in Global Governance, 54 UCLA Law Review 2006-2007, S. 913. 


\section{Marktmechanismus: Netzwerkexternalitäten}

Netzwerkexternalitäten und damit einhergehend die Schaffung von Clubgütern können genutzt werden, um private Akteure und Staaten als Normadressaten zur Einhaltung des Völkerrechts anzuhalten. Clubgüter sind ökonomisch gesprochen diejenigen Güter, bei welchen zwar - wie bei privaten Gütern - Ausschließbarkeit vorliegt, nicht aber rivalisierender Konsum. Rivalisierende, aber nicht ausschließbare Güter sind demgegenüber sog. Allmendegüter, welche in der Regel an Übernutzung leiden, wie etwa Fischereigründe, saubere Luft oder Ölfelder. Regeln gegen die Übernutzung von Allmenden lassen sich nur sehr schwer durchsetzen. Regeln, die Clubgüter regulieren, sind dagegen erheblich einfacher zu effektuieren, da Normverletzer aus dem Club ausgeschlossen werden können und deshalb höhere Anreize zu normkonformem Verhalten bestehen. Der Trick zur Effektuierung des Völkerrechts besteht daher darin, Institutionen zu schaffen, welche öffentliche Güter oder Allmendegüter in Clubgüter umwandeln.

Nehmen wir ein Beispiel aus der Geldwäsche- und Terrorismusfinanzierungsbekämpfung. Die Financial Action Task Force (FATF) wurde durch die G7 Staaten ins Leben gerufen, um soft law-Empfehlungen ${ }^{168}$ für die Geldwäsche- und Terrorismusfinanzierungsbekämpfung auszusprechen. Ziel ist es, die Empfehlungen global durchzusetzen, obwohl die FATF nur 34 Mitgliedstaaten zählt. Staaten, die den Empfehlungen nicht nachkommen, werden auf eine schwarze Liste gesetzt. Das allein würde viele Staaten vermutlich noch nicht stören. Der Mechanismus funktioniert allerdings so, dass die Banken der gelisteten Staaten nur noch unter erheblich erschwerten Bedingungen mit Banken in nicht gelisteten Staaten der G7 (den großen Finanzzentren) kontrahieren können. Mit anderen Worten: Halten sich Staaten nicht an die Normen, werden sie aus dem Club der freien Kapitalmärkte ausgeschlossen; ihre Banken können z.B. keine Dollargeschäfte mehr abwickeln. Die Banken werden ihrerseits ihre Regierungen dazu anhalten, die Empfehlungen der FATF umzusetzen, womit die o.g. politischen Prozesse genutzt werden. Ein anderes Beispiel der Nutzung des „Clubmechanismus“ ist das Basler Übereinkommen über die Kontrolle der grenzüberschreitenden Verbringung gefährlicher Abfälle und ihrer Entsorgung vom 22. März 1989, das in Art. 4 den Abfallhandel mit Nichtvertragsstaaten verbietet.

Bedingungen für das Funktionieren dieses Mechanismus' können der Theorie der Netzwerkexternalitäten entnommen werden. ${ }^{169}$ Für Software und soziale Netzwerke etwa gilt: je größer das Netzwerk, desto höher der individuelle Nutzen des Benutzers

168 Interessanterweise sind viele der Empfehlungen auch kodifiziert in der UN Convention against Transnational Organized Crime (Palermo Convention); A/55/383 of Nov. 2, 2000 sowie in der UN Convention against Illicit Traffic in Narcotic Drugs and Psychotropic Substances; 1988; UN Convention for the Suppression of the Financing of Terrorism, 1999.

169 Grundlegend M.L. Katz/C. Shapiro, Network Externalities, Competition, and Compatibility, 75 American Economic Review 1985, S. 424. 
aufgrund von Skalenerträgen und reduzierten Inkompatibilitäten. Alle Akteure haben in diesen Konstellationen Anreiz dazu, sich an die Normen zu halten; es gibt keine Anreize, zu defektieren, vorausgesetzt, dass ausreichend Information vorliegt. Hier erlangen Monitoring und Reporting Mechanismen besondere Bedeutung. Ist die Information über Normbrecher erst einmal vorhanden, dann ist das System aber selbstdurchsetzend. Dieses Beispiel zeigt, dass globale öffentliche Güter (etwa die Terrorismusbekämpfung) durch geschickte Institutionen in Clubgüter umgewandelt und Normen auf diese Weise effektuiert werden können. Ebenso funktioniert die Zertifizierung, wenn sie über „self-regulation“ läuft (wie etwa bei sektorspezifischen Verbänden). Da hier jedes Mitglied aufgrund des Reputationswertes der Zertifizierung ein Interesse an der Einhaltung hat, sind die peer-monitoring Eigenschaften des Systems in der Regel gut. Zuwiderhandelnde Mitglieder werden schlussendlich aus dem „Club“ ausgeschlossen: „Functionally, enforcement is based on deterrence through signalling rather than via direct punishment... Punishment often constitutes the last resort which arises when the threat to the collective reputation calls for exclusion from the organization or for contractual termination “. ${ }^{170}$ Diese Art des Mechanismus' kann angewendet werden in all jenen Situationen, in denen sich ein öffentliches Gut in ein Clubgut umwandeln lässt.

\section{E. Ausblick: Eine Skizze von Bauprinzipien zur Effektuierung des Völkerrechts}

Das Völkerrecht kennt vielerlei Wege der Effektuierung, mit verschiedenen Akteuren, auf verschiedenen Ebenen und mit verschiedenen Mechanismen. Eine Konzentration nur auf zwischenstaatliche Mechanismen wird den vielen Wegen ebenso wenig gerecht wie eine Konzentration auf Gerichte oder andere Streitbeilegungsmechanismen, auch wenn sie innerstaatlich sind. Völkerrecht wird auch transnational über Konsumentenpräferenzen effektuiert, sei es durch Labelling oder vermittels privatrechtlicher Verträge. Es spielen immer Werte und Interessen zusammen; eine Konzentration nur auf eine der Theorien der internationalen Beziehungen liefe Gefahr, wichtige Mechanismen zu übersehen.

Ein Kasten mit Bauprinzipien zur Effektuierung des Völkerrechts kann hier nur kursorisch skizziert werden. Zunächst sind die grundlegenden Problemkonstellationen aufzuzeigen. Koordinationsverträge werden von Kooperationsverträgen unterschieden. Die Durchsetzung ersterer stößt nicht auf Probleme, weil es im Interesse eines jeden Staates liegt, sich daran zu halten. Schwieriger ist es bei den Kooperationsverträgen, bei denen jeweils unterschiedliche Spiele gespielt werden. Hier kommt es zur Effektuierung maßgeblich darauf an, ob ein Spiel als Vertrauensspiel oder als Gefangenendilemma charakterisiert wird. Während Vertrauensspiele primär der Monitoring und Reporting Mechanismen bedürfen, müssen bei einem Gefangenendi- 
lemma institutionell die Auszahlungen so verändert werden, dass sich ein Rechtsbruch nicht mehr lohnt. Dies kann über die drei genannten Mechanismen der Reputation, der Retaliation und der Reziprozität erfolgen. Allerdings gilt es, hier erneut zu differenzieren. Traité-contrats können über den Reziprozitätsmechanismus zwischenstaatlich effektuiert werden; internationale Gerichte sind hier von besonderer Bedeutung. Der Reputationsmechanismus spielt ebenfalls eine Rolle.

Ein besonderes Problem der Effektuierung ergibt sich aber bei den traité-lois. Hier werden in aller Regel globale öffentliche Güter geschützt wie im Umweltrecht. Das Reziprozitätsprinzip spielt daher keine Rolle, es sei denn, die Einhaltung wird durch Linkages mit anderen Verträgen effektuiert. Zusätzlich können hier Marktmechanismen zum Tragen kommen. Voraussetzung dafür ist allerdings, dass Normbrecher oder Staaten, die nicht ratifizieren, aus dem Club ausgeschlossen werden können. Ebenso lassen sich Konsumentenpräferenzen nutzen, die es erlauben, durch Labelling umweltfeindliche Produkte oder Produktionsmethoden zu kennzeichnen und die so den Konsumenten ermöglichen, über den Markt die Normen zu effektuieren (etwa carbon-footprint labels). Dies ist insbesondere in solchen Konstellationen notwendig, in denen die Regierungen der jeweiligen Staaten - etwa aufgrund starker Lobbygruppen - das Völkerrecht nicht umsetzen. Der Absatzrückgang umweltfeindlicher Produkte kann so zu einer Veränderung des Produzentenverhaltens führen, da durch den Nachfragerückgang die Preise fallen und damit die auch die Anreize, die Norm zu brechen.

In dem ebenfalls mit Durchsetzungsschwierigkeiten behafteten Bereich der Menschenrechte können Individualklagen weiter effektuiert werden mit diversen Maßnahmen, etwa der Zulassung von altruistischen NGO Klagen, mit anderen Formen von Massenklagen oder durch Prozesskostenhilfe. Auch hier ist die Verlinkung mit anderen Verträgen möglich, um den Reziprozitätsmechanismus nutzen zu können. Nicht zuletzt ist auch hier der Marktmechanismus von Bedeutung.

Um den Baukasten auszubauen bedarf es weitergehender Untersuchungen. Zum einen wäre eine ausführliche funktionale Rechtsvergleichung der Durchsetzungsmechanismen des Völkerrechts (inklusive soft law) notwendig. So können etwa verschiedene Umweltschutzverträge miteinander verglichen werden. Ebenso können auch verschiedene regionale Menschenrechtssysteme verglichen werden. Die Unterschiede in den institutionellen Details der Durchsetzungsmechanismen können Aufschluss geben über die jeweilige Effektuierung des Rechts. Zum anderen ist mehr empirische Forschung notwendig und wünschenswert. Nur faktenbasiertes Wissen über die Effektuierung von Völkerrecht erlaubt gutes institutionelles Design. 